\title{
Suppressors of an Arabidopsis thaliana phyB Mutation Identify Genes That Control Light Signaling and H ypocotyl Elongation
}

\author{
Jason W. Reed,* Rangasamy P. Elumalai* and Joanne Chory ${ }^{\dagger}$ \\ *Biology Department, University of N orth Carolina, Chape H ill, North Carolina 27599-3280, and ${ }^{\dagger} \mathrm{H}$ oward $\mathrm{H}$ ughes M edical Institute, \\ Plant Biology Laboratory, The Salk Institute, San Diego, California 92186-5800 \\ Manuscript received August 18, 1997 \\ Accepted for publication November 17, 1997
}

\begin{abstract}
ABST RACT
Ambient light controls the development and physiology of plants. The A rabidopsis thaliana photoreceptor phytochrome $\mathrm{B}$ (PHYB) regulates developmental light responses at both seedling and adult stages. To identify genes that mediate control of development by light, we screened for suppressors of the long hypocotyl phenotype caused by a phyB mutation. Genetic analyses show that the shy (short hypocotyl) mutations we have isolated fall in several loci. Phenotypes of the mutants suggest that some of the genes identified have functions in control of light responses. Other loci specifically affect cell elongation or expansion.
\end{abstract}

$P$ LANTS adjust their development in response to ambient wind, temperature, water, and light. Such adjustments allow plants to grow in a variety of sites and to adapt to seasonal changes in external conditions. Light isamong the most relevant environmental signals because plants use light for photosynthesis and because light conditions reflect both the local growth environment and diurnal and seasonal time (Smith 1994). Plants sense the light environment using a battery of photoreceptors that specifically control development. These include red/ far-red light photoreceptors called phytochromes, blue light photoreceptors called cryptochromes, and unnamed photoreceptors that mediate phototropism and UV light responses (von Arnim and Deng 1996; Fankhauser and Chory 1997).

The phytochromes are the most extensively characterized developmental photoreceptors in plants (Quail 1991; Fu r u ya 1993). They are soluble dimeric proteins, and each $\sim 120-k D$ monomer has a covalently attached linear tetrapyrrole chromophore. Phytochromes are synthesized in the dark in a red light-absorbing form called Pr. U pon absorption of red light, they are converted to a far-red light absorbing form called Pfr. On the basis of physiological, genetic, and biochemical studies, Pfr is thought to be the active form. Studies of seed germination have suggested that Pr may also have an activity that counteracts the activity of the Pfr form (Reed et al. 1994; Sh inomura et al. 1994).

Although the mechanisms of phytochrome signal transduction are uncertain, several models have been proposed. A cyanobacterial phytochrome homolog signals by a phosphorelay mechanism (Yeh et al. 1997),

Correspon ding author: Jason W. Reed, Department of Biology, U niversity of North Carolina, CB \#3280, Coker Hall, Chapel Hill, NC 275993280. E-mail: jreed@email.unc.edu suggesting that higher plant phytochromes might also signal in this fashion. Pharmacological studies have suggested that phytochromes may act through branched signaling pathways involving $G$ proteins, cyclic GMP, and calcium (Sh ackl ock \& al. 1992; N eu h aus et al. 1993; Bow ler et al. 1994). More recently, it has been reported that phytochrome B migrates to the nucleus in the light, suggesting that phytochromes may signal in the nucleus as well as in the cytoplasm (Sakamo to and Nagat ani 1996).

The genetics of plant light responses have been studied most extensively in A rabidopsis thaliana. Arabidopsis has five genes that encode phytochrome apoproteins (Sharrock and Quail 1989; Clack et al. 1994), and mutations are known in three of these, PHYA (D eh esh et al. 1993; Whit el am et al. 1993; Reed et al. 1994), PH YB (Koornneef et al. 1980; Reed et al. 1993), and PHYD (Auker man et al. 1997). Analyses of the phenotypes of these mutants have shown that these three phytochromes each mediate overlapping subsets of light responses, but often do so under distinct light conditions. For example, phyA mutant seeds germinate poorly in response to very low fluence light over a wide spectral range (Bot to et al. 1996; Sh inomura et al. 1996), whereas phyB mutant seeds germinate poorly in response to red light (Reed et al. 1994; Shinomura et al. 1994, 1996). phyA mutants fail to inhibit hypocotyl elongation in response to far-red light ( $\mathrm{N}$ agat ani et al. 1993; Parks and Quail 1993; Whit el am et al. 1993), whereas phyB mutants fail to inhibit hypocotyl elongation in response to red light (Koornneef et al. 1980). Finally, phyA mutants flower later than wild-type plants in response to night breaks or day length extensions (Johnson et al. 1994; Reed et al. 1994), whereas phyB mutants flower earlier than wild-type plants under a variety of conditions ( $G$ ot 0 et al. 1991; Reed et al. 1993; 
Bagnal I etal. 1995; Koornneef et al. 1995). Effects of phyA and phyD mutations on the inhibition of hypocotyl elongation by red light can be seen in a phyB mutant background (Reed et al. 1994; Auker man et al. 1997). These results reveal that the multiplicity of phytochromes serves in part to increase the versatility of the plant in responding to different light environments. These phenotypic analyses al so suggest that the signaling pathways initiated by the different phytochromes may overlap. hy4 mutants deficient in the blue light photoreceptor cryptochrome 1 have some phenotypes in common with the phytochrome-deficient mutants (Koornneef et al. 1980; Young et al. 1992; Ahmad and Cashmore 1993), suggesting that blue light signaling path ways also converge with phytochrome signaling path ways. Physiological analyses of photoreceptor mutants have also suggested that blue and red light systems interact functionally (Casal and Boccal andro 1995; Ahmad and Cash more 1997).

Mutations that identify possible downstream components of phytochrome signaling have been isolated in long hypocotyl screens, in screens for early flowering mutants, and in screensfor seedlingswith characteristics of light-grown plants in the dark. The fhyl and fhy3 mutants have long hypo cotyls in far-red light, suggesting that they may have lesions in a PH YA-specific signaling pathway (Wh it el am et al. 1993; Bar nes et al. 1996). hy5 mutants have long hypocotyls under all light conditions, suggesting that $\mathrm{HY} 5$ may act downstream of the convergence of different photoreceptor pathways ( Ko o r n n eef et al. 1980). Consistent with this idea, H Y 5 encodes a basic leucine zipper transcription factor ( 0 yama et al. 1997). elf3 mutants have an elongated hypocotyl under all light conditions, and they flower early (Zagot t a et al. 1996). The cr88, pef1, pef2, pef3, and red1 mutants have long hypocotyls in red light ( $c r 88$, pef2, pef3, and red1) or in both red and far-red light (pef1; A h mad and Cashmore 1996; L in and Cheng 1997; Wagner et al. 1997). These genes may encode positive regulators of light signaling.

Screens for mutants with short hypocotyls and leaf development in the dark have yielded candidate negative regulators. A series of cop, det, and fus mutants have short hypocotyls, develop leaves, and express lightinduced genes in the dark (reviewed in Wei and Deng 1996; Fankhauser and Ch or y 1997). The biochemical function sof the products of these genes remain unclear. However, the DET1, COP1, COP9, and FUS6 proteins localize to the nucleus, suggesting that they may repress gene expression in the dark (Pepper et al. 1994; von Arnim and Deng 1994; Chamovitz et al. 1996; St aub et al. 1996). Double-mutant plants that carry photoreceptor mutations and det/cop/ fus mutations have phenotypes consistent with the DET/COP/ FUS gene products functioning downstream of photoreceptor pathways (Chory 1992; Ang and Deng 1994; M isér a et al. 1994; Wei et al. 1994a,b). More detailed analysis of cop/ det/ fus mutant phenotypes suggests that these genes may play a more general role in regulating gene expression in response to a variety of stimuli in addition to light. For example, detl seedlings express L H CB (encoding light harvesting chlorophyll a/ b-binding protein) and other genes in roots (Ch o r y and Pet 0 1990); and cop1, det1, and cop 9 mutants overexpress genes that are normally activated by pathogen infection, hypoxia, or developmental signals, as well as genes normally activated by light (M a yer et al. 1996). Finally, several other mutants with subsets of the phenotypes described above have been described, including det2, det3, cop2, cop3, cop4, and docl (Chory et al. 1991; Cabrera y Poch et al. 1993; H ou et al. 1993; Li et al. 1994).

Although many genesinvolved in light signaling have been identified in these screens, it is likely that numerousother relevant loci remain to be discovered. In other systems, screens for suppressors and enhancers of mutations in a pathway have identified important new genes (for example, Kar im et al. 1996). These genetic methodologieshave been used lessfrequently in plants (Ko or nneef et al. 1982; Niyogi et al. 1993; Carol et al. 1995; Cer nac et al. 1997; Sil ver st one et al. 1997), but promise to become very useful for dissecting light responses. A screen for suppressors of a hy2 phytochrome chromophore-deficient mutation (Kim et al. 1996) and a screen for suppressors of a dell mutation (Pepper and Ch ory 1997) have recently revealed more candidate light-signaling mutations. To identify other genes involved in light signaling, we have conducted a screen for mutations that suppress the long hypocotyl phenotype caused bya phyB mutation. In this report, we describe the results of our initial screen. Following the precedent established by Kim et al. (1996), we have called these new mutations shy (for short hypocotyl and suppressor of hy). We have characterized various phenotypes of the mutants, allowing us to identify those that affect light signaling.

\section{MATERIALS AND METHODS}

Mutagenesis and genetic methods: Mutant phyB-1 (previously called hy3-B 064 ) is in the Landsberg erecta background and has a stop codon in the PH YB coding sequence (Ko or nneef et al. 1980; Reed et al. 1993; Q uail et al. 1994). We incubated 5000 phyB-1 M 1 seeds overnight in $0.3 \%$ ethyl methane sulfonate and then rinsed several times with water. We collected M2 progeny in eight batches from $\sim 600 \mathrm{M} 1$ plants per batch. We screened $\sim 14,000$ M2 seedlings on MS/ sucrose/ agar plates ( see below) under white light for short hypocotyl variants. To detect revertants of the starting phyB-1 mutation and to follow the phyB-1 mutation in subsequent genetic manipulations, we assayed for the presence (wild-type allele) or absence ( phyB-1 mutant allele) of an AlwN I restriction site in a PH YB-specific PCR product amplified from chromosomal DNA of the plant being tested (Reed et al. 1993). Mutants were judged to be independent if they came from different batches or if they had clearly distinguishable phenotypes.

To separate the shy mutation from the starting phyB-1 mutation, we crossed the phyB-1 shy strains to wild-type Landsberg 
erecta, allowed these $F_{1}$ plants to self-fertilize, and identified $\mathrm{PH} Y \mathrm{~B} / \mathrm{PH}$ YB shy/ shy progeny in the $\mathrm{F}_{2}$ or $\mathrm{F}_{3}$ generation. For shy mutations that confer an obvious phenotype (such as dwarfism) in a wild-type background we identified PHYB/ $\mathrm{PHYB}$ shy/ shy plants in the $\mathrm{F}_{2}$ generation. For mutations that confer a phenotype similar to the wild-type phenotype in a phyB-1 mutant background and that confer no dramatic phenotype in the wild-type PHYB background, we identified $\mathrm{PH} Y \mathrm{~B} /$ phyB-1 heterozygous $\mathrm{F}_{2}$ plants from the outcross to wild type that did not segregate tall (phyB-1/ phyB-1 SH Y/SH Y or phyB-1/ phyB-1 SH Y/ shy) $F_{3}$ progeny. The failure to segregate tall $F_{3}$ plants indicated that these $F_{2}$ plants were homozygous for the shy mutation (or that the shy mutation was linked to the phyB-1 mutation, a possibility tested in the mapping experiments described below). From the $\mathrm{F}_{3}$ progeny populations, we identified PH YB/ PH YB individuals of gen otype PH YB/ $P H Y B$ shy/ shy. In these genetic manipulations, we distinguished the PHYB and phyB-1 alleles by the PCR-based assay described above.

We mapped the shy mutations using PCR-based SSLP and CAPS markers polymorphic between Landsberg and Columbia ecotypic backgrounds (Konieczny and Ausubel 1993; Bell and Ecker 1994). We crossed phyB-1/ phyB-1 shy/ shy plants (Landsberg erecta background) with phyB-9/ phyB-9 plants (Columbia background), and assayed DNA from individual $F_{2}$ progeny for Landsberg- or Columbia-specific polymorphisms. Both the phyB-1 and phyB -9 mutations create stop codons in the PH YB-coding sequence (R eed et al. 1993). Since suppressor mutations isolated in this screen should bypass the requirement for $\mathrm{PHYB}$, we expected the phyB-1 and phyB-9 alleles to behave equivalently in the mapping populations. In cases where we suspected that a mutation was allelic to a previously known mutation, we performed complementation tests between the shy mutation and the previously described mutation, and we looked for lack of segregation of $F_{2}$ plants with a wild-type phenotype, indicating that the two mutations mapped to the same location. In cases where we found allelism to a previous locus, we have given the new mutation an allele designation that incorporates the established gene name.

Phenotypic tests: Seedswere surface sterilized and plated on Murashige and Skoog (MS)/ agar plates [ $1 \times$ MS salts ( GIBCO, Grand Island, NY), 0.8\% phytagar (GIBCO), 1× Gamborg's B5 vitamin mix (Sigma, St. Louis, MO)] with or without $2 \%$ sucrose, stored overnight at $4^{\circ}$, and moved to the appropriate light condition. For hypocotyl length tests in red light, we used LED red light sources emitting light with a peak at 670 $\mathrm{nm}$ and a half bandwidth of $25 \mathrm{~nm}$ (Quantum Devices, Inc., Barneveld, WI). For far-red light, we used LED sources emitting light with a peak at $730 \mathrm{~nm}$ and a half bandwidth of 25 nm (Quantum Devices). For blue light, we used cool white fluorescent bulbs filtered through Schott blue glass filter No. 5-57 (N ewport Industrial Glass, Costa M esa, CA). For fluence rate/ response experiments, light was filtered through various thicknesses of bronze plexiglass No. 2412 (Golden Rule Plastics, $\mathrm{H}$ aw River, NC). This filter causes minimal distortion of the light spectrum (data not shown). Light levels were measured with an LI-189 quantum radiometer (Li-Cor, Lincoln, $N E$ ), or extrapolated based on numbers of layers of plexiglass. For red and far-red fluence rate-response experiments, we grew seedlings on MS/ agar plates (without sucrose) that were placed vertically behind various thicknesses of plexiglass, with the light source placed so as to project horizontally. After 5 days, we took digital images of the plates with a CCD camera and measured the hypocotyl lengths using image analysis software (NIH Image, Bethesda, MD). For root length experiments, we grew seedlings in red light $\left(30-50 \mu \mathrm{mol} \cdot \mathrm{m}^{-2}\right.$ - $\mathrm{sec}^{-1}$ ) on vertical MS/ sucrose/ agar plates for 5 days and measured roots against a ruler. In other experiments and for

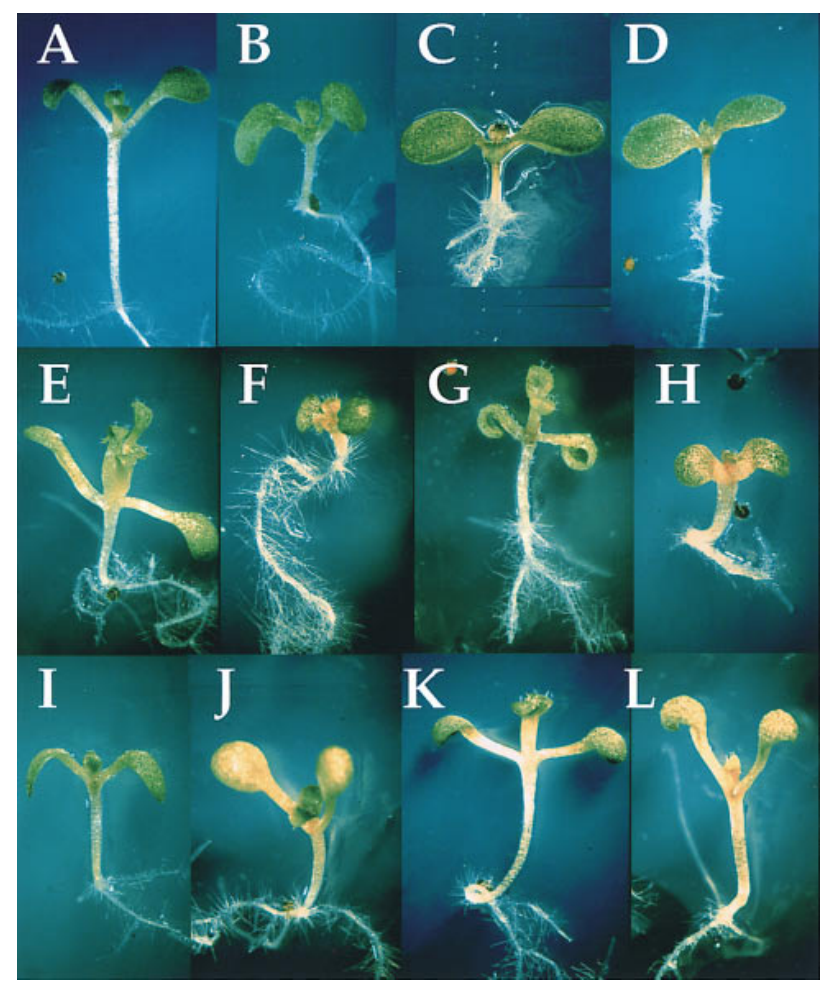

Figure 1.- phyB-1 shy seedlings grown in white light. Seedlings were grown for 8 days on MS/ sucrose plates. Genotypes shown are (A) phyB-1 (starting strain), (B) Landsberg erecta (wild-type parent), (C) phyB-1 shy2-2, (D) phyB-1 shy2-3, (E) phyB-1 amp1-4, (F) phyB-1 shy-115, (G) phyB-1 pom1-15, (H) phyB-1 bot1-5, (I) phyB-1 shy3-1, (J) phyB-1 shy4-1, (K) phyB-1 shy5-1, and (L) phyB-1 shy6-1. shy4-1 and shy4-2 seedlings appeared similar to each other, and pom1-14 and pom1-15 seedlings appeared similar to each other. Therefore, only one mutant at each locus is shown.

all blue light experiments, we grew plants on plates placed horizontally and measured hypocotyl lengths against a ruler.

For flowering time determinations, we grew seedlings on MS/ sucrose/ agar plates for 10-14 days and then transplanted them to soil (Pro-Mix BX; Hummert, St. Louis, MO). Experiments were performed in a Conviron growth chamber at $21^{\circ}$. Light was provided on a $9 \mathrm{~h}: 15 \mathrm{~h}$ day:night cycle from 12 fluorescent (F72T 12/ CW/ VH O, 160 W) and six incandescent ( $60 \mathrm{~W})$ bulbs, and had an intensity at plant height of 100-230 $\mu \mathrm{mol} \cdot \mathrm{m}^{-2} \cdot \mathrm{sec}^{-1}$, depending on the experiment. We repeated the experiment six times, testing each genotype in between one and five different experiments (average $=2.5$ ).

\section{RESULTS}

Isolation and genetic analysis of shy mutants: From our screen, we isolated 13 independent shy ( short hypocotyl, or suppressor of hy) variantswith mutationsat sites distinct from the starting phyB mutation (see mat er ial s and methods). As described below, we have named those that turned out to be alleles of previously known loci according to the established gene names. Figure 1 shows phyB-1 shy mutant seedlings grown for 8 days in white light. They fall into several distinct phenotypic 
TABLE 1

Classes of shy mutations

\begin{tabular}{llll}
\hline Mutation & \multicolumn{1}{c}{ Phenotype } & Dom./ rec. $^{\text {a }}$ & Allelism $^{\text {b }}$ \\
\hline shy2-2 & Leaves curl upward & Semi-D & shy2-1 \\
shy2-3 & Leaves curl upward & Semi-D & shy2-2 \\
amp1-4 & Extra leaf production & $r$ & amp/pt \\
shy-115 & Brassinosteroid-deficient dwarf & $r$ & \\
shy-802 & Brassinosteroid-deficient dwarf & $r^{c}$ & \\
pom1-14 & Crooked hypocotyl in dark & Semi-D & pom1-7 \\
pom1-15 & Crooked hypocotyl in dark & $r$ & pom1-14 \\
bot1-5 & Short, round tissues & $r$ & botero1-1 \\
shy3-1 & Quantitative changes & Semi-D & \\
shy4-1 & Quantitative changes & $r$ & shy4-2 \\
shy4-2 & Quantitative changes & $r$ & shy4-1 \\
shy5-1 & Quantitative changes & $r$ & \\
shy6-1 & Quantitative changes & $r$ & \\
\hline
\end{tabular}

a Dominance, semidominance, or recessiveness for hypocotyl length in red light (Table 2).

${ }^{b}$ Indicated are mutations found to be allelic to shy mutations on the basis of complementation and mapping data (see text). In addition, we have recently obtained DNA sequence evidence that the partially dominant mutations shy2-1, shy2-2, and shy2-3 each have a mutation in the SH Y 2 gene ( Q. Tian and J. W. Reed, unpublished result).

'Although we did not analyze the dominance or recessiveness of shy-802 quantitatively (Table 2), observation of $F_{1}$ seed of a backcross indicated that the dwarf phenotype was substantially if not completely recessive (data not shown).

classes, as summarized in Table 1 and described in more detail below.

To assess the degree of dominance or recessiveness of the shy mutations in the phyB-1 mutant background, we measured hypocotyl lengths after growth in constant red light, as this parameter is sensitive and easy to score. We compared hypocotyl length s of phyB-1/ phyB-1 SHY/ shy $F_{1}$ plants with those of the phyB-1/ phyB-1 SH Y/SH Y and phyB-1/ phyB-1 shy/ shy parents. As shown in Table 2, the amp1-4, shy-115, shy-802, pom1-15, bot1-5, shy4-1, shy4-2, shy5-1, and shy6-1 heterozygous seedlings were the same height as SH Y / SH Y seedlings, indicating that these mutations are each recessive for hypocotyl length. In contrast, the shy2-2, shy2-3, pom1-14, and shy3-1 heterozygous seedlings were significantly shorter than the $\mathrm{SH} Y$ / SH Y seedlings and significantly taller than the corresponding shy/ shy seedlings, indicating that these mutations are each partially dominant.

To determine whether the short hypocotyl phenotype of these mutants was caused by mutation at a single locus, we checked the segregation of the short hypocotyl phenotype in the $F_{2}$ generation of these backcrosses. For each mutant, the phenotype segregated in a manner consistent with a mutation at a single locus (Table 3).

We found three allelic pairs in our screen, at the SH Y 2, POM 1, and SH Y 4 loci. As the shy2 mutations are partially dominant, our assessment that they are alleles of SH Y 2 (and of each other) is based on their conferring similar phenotypes as shy2-1 does (Kim et al. 1996), as well as mapping to the same location (Table 4). In addi- tion, we have recently obtained independent molecular evidence that shyz-1, shyz-2, and shyz-3 are allelic $(Q$. Tian and J. W. Reed, unpublished data). As we identified single mutations at the remaining seven loci described here, the screen has not been saturated, and there are probablyseveral other loci that can be mutated to give a shy phenotype.

We mapped the shy mutations by outcrossing them to a different ecotype and assaying polymorphic markers or byestablishing allelism with previously mapped mutations ( see mat erial s and met hods). Mapping results are summarized in Table 4. We obtained linkage to a polymorphic marker for all the loci except for the shy5-1 mutation, for which different mapping populations failed to show consistent linkage (data not shown). The 13 mutationsfall in three previouslyknown genes, AM P, SH Y2, and POM 1 (Chaudhury et al. 1993; H auser et al. 1995; Kim et al. 1996); two probable DWF genes (mutated in shy- 115 and shy-802, but not assigned by complementation-see below; Fel d mann et al. 1989); and five new genes, BOT 1 (BOTER 01; H. H öft e, personal communication), $\mathrm{SH} Y 3, \mathrm{SH} Y 4, \mathrm{SH} Y 5$, and $\mathrm{SH} Y 6$.

To determine whether any of the shy mutations completely quench PHYB signaling, we tested whether the shy mutations were epistatic to the phyB-1 mutation. We compared the hypocotyl lengths of phyB-1 shy double mutants with that of the phyB-1 single mutant and with those of the corresponding shy single mutants. For each mutant, we identified PH YB shy plants among progeny of an outcross to a wild-type Landsberg erecta plant ( see 
TABLE 2

Hypocotyl lengths of phyB-1/ phyB-1 shy/ shy and phyB-1/phyB-1 SH Y/ shy seedlings in red light

\begin{tabular}{|c|c|c|c|c|c|c|c|c|c|c|}
\hline \multirow[b]{2}{*}{ shy mutation } & \multicolumn{5}{|c|}{ shy/ shy seedlings } & \multicolumn{5}{|c|}{ SH Y/ shy seedlings } \\
\hline & $x$ & $\sigma$ & $n$ & $t^{b}$ & $\mathrm{~Pb}$ & $x$ & $\sigma$ & $n$ & $t^{c}$ & $\mathrm{P}^{\mathrm{c}}$ \\
\hline shy2-2 & 2.5 & 0.4 & 20 & 13.9 & $<0.001$ & 4.1 & 0.3 & 20 & 17.1 & $<0.001$ \\
\hline shy2-3 & 4.2 & 1.0 & 20 & 10.12 & $<0.001$ & 7.4 & 0.8 & 16 & 6.50 & $<0.001$ \\
\hline ampl-4 & 4.4 & 1.1 & 20 & 10.4 & $<0.001$ & 9.7 & 2.2 & 20 & 0.51 & $>0.5$ \\
\hline shy-115 & 4.0 & 1.0 & 20 & 9.89 & $<0.001$ & 8.9 & 1.3 & 7 & 1.71 & $<0.1$ \\
\hline pom1-14 & 2.2 & 0.4 & 40 & 12.0 & $<0.001$ & 7.5 & 2.7 & 22 & 6.07 & $<0.001$ \\
\hline pom1-15 & 2.6 & 0.7 & 20 & 16.47 & $<0.001$ & 10.1 & 1.7 & 10 & 0.40 & $>0.5$ \\
\hline bot1-5 & 2.6 & 0.6 & 40 & 13.3 & $<0.001$ & 9.8 & 3.3 & 26 & 0.25 & $>0.5$ \\
\hline shy3-1 & 6.5 & 1.0 & 20 & 3.98 & $<0.001$ & 8.2 & 1.6 & 22 & 4.80 & $<0.001$ \\
\hline shy4-1 & 5.8 & 1.7 & 20 & 5.28 & $<0.001$ & 9.3 & 2.3 & 19 & 1.48 & $<0.2$ \\
\hline shy4-2 & 7.2 & 1.4 & 19 & 3.77 & $<0.001$ & 10.7 & 3.4 & 10 & 1.40 & $<0.2$ \\
\hline shy5-1 & 6.8 & 1.7 & 40 & 2.46 & $<0.025$ & 9.6 & 6.9 & 40 & 0.47 & $>0.5$ \\
\hline shy6-1 & 8.2 & 1.3 & 20 & 4.04 & $<0.001$ & 10.4 & 1.9 & 17 & 1.23 & $<0.4$ \\
\hline
\end{tabular}

${ }^{\text {a }} \mathrm{H}$ ypocotyl lengths in millimeters.

${ }^{b}$ t-test and $P$ value from comparing the phyB-1/phyB-1 shy/ shy and phyB-1/phyB-1 SH Y/ shy seedling hypocotyl lengths. The low $P$ values indicate that for all of the mutations, the hypocotyl length of the shy/ shy homozygote is significantly shorter than that of the $\mathrm{SH} Y /$ shy heterozygote, and that therefore none of the shy mutations is completely dominant.

' t-test and P value from comparing the phyB-1/ phyB-1 SH Y/SH Y and phyB-1/ phyB-1 SH Y/ shy seedling hypocotyl lengths. For the phyB-1/phyB-1 SH Y/SH Y single mutant, the hypocotyl measurements were $x=9.9, \sigma=1.5$, and $n=140$. The results indicate that only shy3-1, pom1-14, shy2-2, and shy2-3 heterozygotes are significantly shorter than the phyB-1/phyB-1 single mutant and, therefore, are not recessive.

materials and methods). Table 5 shows hypocotyl lengths in red light of each of the PH YB shy and phyB-1 shy seedlings. In most cases, the phyB-1 shy double mutant is significantly taller than the PH YB shy single mutant, indicating lack of epistasis (Table 5). For pom1-14, the difference between PHYB and phyB-1 genotypes was not significant, indicating that pom1-14 is epistatic to phyB-1 for this phenotype.

Phenotypic analyses of shy mutant plants: To assess whether the shy mutation saffect genes involved in PHYB signal transduction or, more generally, in light signaling, we checked several phenotypes. Because a number of photomorphogenic mutations affect dark growth, we examined the morphology of shy seedlings in the dark. We also examined whether the shy mutation saffect lightdependent phenotypes. As described above, phyB mutants have long hypocotyls in red light, have short roots, and flower early. We expected that mutations specifically limiting hypocotyl cell enlargement or elongation would suppress onlythe long hypocotyl phenotype (and perhaps other elongation phenotypes such as elongated root hairs or bolting stems), but not the flowering time or short root phenotypes. In contrast, mutations that affect a general control function might suppress multiple phenotypes caused by the phyB-1 mutation. These phenotypic criteria have indeed allowed us to distinguish mutations that affect elongation from those that affect putative control functions. Within this broad classification, many of the mutants have unique characteristics that define distinct roles in development (Table 1).
TABLE 3

Segregation of shy mutant phenotypes among $F_{2}$ progeny of backcrosses

\begin{tabular}{lcccc}
\hline shy mutation & $\begin{array}{c}\text { Tall } \\
\text { progeny }\end{array}$ & $\begin{array}{c}\text { Short } \\
\text { progeny }\end{array}$ & $\chi^{2 b}$ & $P$ \\
\hline shy2-2 & 34 & 89 & 0.46 & $>0.25$ \\
shy2-3 & 52 & 163 & 0.08 & $>0.5$ \\
amp1-4 & 146 & 52 & 0.17 & $>0.5$ \\
shy-115 & 133 & 48 & 0.22 & $>0.5$ \\
shy-802 & 50 & 16 & 0.02 & $>0.5$ \\
pom1-14 & 47 & 22 & 1.74 & $>0.1$ \\
pom1-15 & 34 & 11 & 0.01 & $>0.5$ \\
bot1-5 & 365 & 104 & 2.00 & $>0.1$ \\
shy3-1 & 45 & 100 & 2.82 & $>0.05$ \\
shy4-1 & 78 & 23 & 0.27 & $>0.5$ \\
shy4-2 & 381 & 136 & 0.47 & $>0.25$ \\
shy5-1 & 96 & 39 & 1.09 & $>0.25$ \\
shy6-1 & 104 & 35 & 0.01 & $>0.5$ \\
\hline
\end{tabular}

a Tall and short $F_{2}$ progeny were distinguished by eye in populations grown in dim white light. For those mutations with quantitative effects ( shy3-1, shy4-1, shy4-2, shy5-1, and shy6-1), we measured hypocotyl lengths of representative $F_{2}$ seedlingsand found that the two visible groups (tall and short) formed a frequency distribution with two peaks, with no seedlins of intermediate hypocotyl length.

${ }^{b} \chi^{2}$ values were calculated for the null hypothesis of 3 tall:1 short segregation, except for the semidominant or dominant mutations shy3-1, shy2-2, and shy2-3, for which the null hypothesis was 1 tall:3 short. 
TABLE 4

Mapping of shy loci

\begin{tabular}{|c|c|c|c|}
\hline shy mutation & Marker & $\begin{array}{l}\text { Recombinant } \\
\text { chromosomes }\end{array}$ & Deduced map position \\
\hline shy2-2 & $\begin{array}{l}\text { PVV4 } \\
\text { nga63 } \\
\text { shy2-1 }\end{array}$ & $\begin{array}{r}15 / 320 \\
8 / 320 \\
0 / 102^{a}\end{array}$ & $\begin{array}{l}\text { Chr. } 1,5 \text { cM below PVV4, } \\
3 \text { cM above nga } 63 \text {, close } \\
\text { to shy2-1 }\end{array}$ \\
\hline shy2-3 & shyz-2 & $0 / 109^{a}$ & Chr. 1, close to shy2-2 \\
\hline amp1-4 & $\mathrm{pt}^{\mathrm{b}}$ & b & Chr. 3, position 81 \\
\hline shy-115 & GL 1 & 19/92 & Chr. 3, 21 cM from GL 1 \\
\hline shy-802 & BGL 1 & $3 / 56$ & Chr. 3, 5 cM from BGL 1 \\
\hline pom1-14 & $\begin{array}{l}\text { PVV4 } \\
\text { NCC1 }\end{array}$ & $\begin{array}{l}4 / 60 \\
5 / 60\end{array}$ & $\begin{array}{c}\text { Chr. 1, } 8 \mathrm{cM} \text { below PVV4, } \\
8 \mathrm{cM} \text { above NCC1 }\end{array}$ \\
\hline pom1-15 & $\begin{array}{l}\text { PVV4 } \\
\text { NCC1 }\end{array}$ & $\begin{array}{l}6 / 60 \\
5 / 60\end{array}$ & \\
\hline bot1-5 & $\mathrm{ADH}$ & $18 / 300$ & Chr. 1, $6 \mathrm{cM}$ below ADH \\
\hline shy3-1 & $\begin{array}{l}\mathrm{m} 323 \\
\mathrm{~m} 429\end{array}$ & $\begin{array}{c}6 / 76 \\
56 / 840\end{array}$ & $\begin{array}{l}\text { Chr. 2, } 8 \mathrm{cM} \text { below m323, } \\
5 \mathrm{cM} \text { below m429 }\end{array}$ \\
\hline shy4-2 & $\begin{array}{l}\text { nga8 } \\
\text { GA1 }\end{array}$ & $\begin{array}{r}14 / 58 \\
8 / 58\end{array}$ & $\begin{array}{l}\text { Chr. 4, } 24 \text { cM below nga } 8 \text {, } \\
14 \text { cM above GA } 1\end{array}$ \\
\hline shy6-1 & $\begin{array}{l}\text { PVV4 } \\
\text { NCC1 }\end{array}$ & $\begin{array}{r}11 / 60 \\
0 / 52\end{array}$ & Chr. 1, close to NCC1 \\
\hline
\end{tabular}

${ }^{a}$ For mapping semidominant shy2 alleles relative to each other, we crossed two shy2 mutants, crossed the resulting $F_{1}$ double heterozygote with wild type, and looked for phenotypically wild-type segregants among the $F_{1}$ progeny of the outcross to wild type. Also included in the shy2-2 $\times$ shy2 -3 data are $1065 F_{2}$ progeny, among which we observed no wild-type plants. This is equivalent to observing $\sqrt{1065}=33$ chromosomes in this cross of dominant mutations.

${ }^{b}$ pt is an allele of amp in the Landsberg erecta background. amp1-4 failed to complement pt, and no wildtype plants were observed among several hundred $\mathrm{F}_{2}$ progeny. Chr., chromosome.

We describe the mutants briefly here to help clarify the presentation below.

The shy2-2 and shy2-3 mutants have leaves that curl up at the edges. The shy2-2 and shy2-3 mutations were each semidominant (Table 2). In these respects, these mutants resemble the shyl and shy2-1 mutants isolated as suppressors of a hy2 mutation (Kim \& al. 1996) and the axr3 mutants isolated as having auxin-resistant root

TABLE 5

H ypocotyl lengths of phyB-1 shy and PH YB shy seedlings in red light ${ }^{\mathrm{a}}$

\begin{tabular}{|c|c|c|c|c|c|c|c|c|}
\hline \multirow[b]{2}{*}{ shy mutation } & \multicolumn{3}{|c|}{ PHYB shy seedlings } & \multicolumn{3}{|c|}{ phyB-1 shy seedlings } & \multirow[b]{2}{*}{$t^{b}$} & \multirow[b]{2}{*}{$\mathrm{P}^{\mathrm{b}}$} \\
\hline & $x$ & $\sigma$ & $\mathrm{n}$ & $x$ & $\sigma$ & $\mathrm{n}$ & & \\
\hline shy2-2 & 2.0 & 0.2 & 40 & 2.6 & 0.2 & 8 & 7.58 & $<0.001$ \\
\hline shyz-3 & 2.1 & 0.5 & 30 & 4.8 & 1.8 & 23 & 7.68 & $<0.001$ \\
\hline amp1-4 & 2.4 & 0.7 & 8 & 4.6 & 0.9 & 14 & 5.68 & $<0.001$ \\
\hline shy-115 & 1.2 & 0.3 & 10 & 2.0 & 0.6 & 6 & 3.31 & $<0.005$ \\
\hline pom1-14 & 2.3 & 0.8 & 27 & 2.4 & 0.5 & 29 & 0.55 & $>0.5$ \\
\hline pom1-15 & 2.5 & 0.7 & 27 & 3.2 & 0.7 & 26 & 3.57 & $<0.001$ \\
\hline botl-5 & 1.7 & 0.5 & 37 & 2.5 & 0.8 & 37 & 4.88 & $<0.001$ \\
\hline shy3-1 & 2.5 & 0.5 & 18 & 4.7 & 1.0 & 21 & 8.25 & $<0.001$ \\
\hline shy4-1 & 2.4 & 0.7 & 15 & 5.6 & 1.4 & 15 & 7.65 & $<0.001$ \\
\hline shy4-2 & 1.8 & 0.6 & 18 & 4.2 & 1.2 & 17 & 7.33 & $<0.001$ \\
\hline shy5-1 & 3.1 & 1.3 & 16 & 5.1 & 1.3 & 9 & 3.54 & $<0.005$ \\
\hline shy6-1 & 2.1 & 0.9 & 11 & 3.7 & 1.1 & 16 & 3.84 & $<0.001$ \\
\hline
\end{tabular}

${ }^{a} \mathrm{H}$ ypocotyl lengths in millimeters. The red light level was $31-37 \mu \mathrm{mol} \cdot \mathrm{m}^{-2} \cdot \mathrm{sec}^{-1}$. Data for bot1-5, pom114 , shy2-2, shy2-3, and pom1-15 were combined from two experiments. All phyB-1 shy seedlings were significantly shorter than phyB-1 seedlings (data not shown).

${ }^{b}$ t-test and $P$ values calculated for comparison of phyB-1 shy hypocotyl lengths with corresponding PH YB shy hypocotyl lengths. 
growth (Leyser et al. 1996). The shy2-2 mutation conferred more extreme phenotypes than the shy2-3 mutation (see below), suggesting that it is the stronger allele. As described below, these mutants may identify an important control function in light-regulated development.

Mutations in the AMP (altered meristem program) gene confer altered phyllotaxy and partially de-etiolated growth in the dark (Chaudhury \& al. 1993; ChinAt kins et al. 1996). Like the previously described amp mutants, the amp1-4 mutant we isolated appears d warfed and has pale leaves and decreased apical dominance. In the light, ampl-4 seedlings formed more leaves than wild-type plants (Figure 1). In the dark, they showed partial leaf development (see below).

The shy-115 and shy-802 mutants are brassinosteroiddeficient dwarfs. These resemble the previously characterized bri1, cbb, det2, dim, and dwf mutants in having small dark green leaves (Fel dmann et al. 1989; Chory et al. 1991; Takahashi et al. 1995; Cl ouse et al. 1996; Li et al. 1996; Szeker es et al. 1996). Such mutants have been shown to have deficiencies in brassinosteroid synthesis or response. Both shy-115 and shy-802 mutants responded to exogenous brassinolide, suggesting that they are deficient in brassinosteroid synthesis (data not shown) . shy-115 plants were fully fertile, whereas shy- 802 plants were almost sterile and produced few seed. Both shy-115 and shy-802 complemented det2-1, and they also complemented each other. These equations therefore represent distinct loci. On the basis of their map positions, shy-115 may be allelic with DWF1 (also called DIM ), and shy-802 may be allelic with DWF4. Because we obtained few shy- 802 mutant seeds, it was difficult to subject the shy-802 mutant to extensive phenotypic analyses. Moreover, light-related phenotypes of brassinosteroid-deficient mutants have been described extensively. Therefore, we omitted the shy- 802 mutant from many of the experiments described below.

pompom1 (pom1) mutants were first isolated as having abnormal root elongation ( $\mathrm{H}$ au ser et al. 1995). The pom1-14 and pom1-15 mutants have similarly deficient root growth (see below). These mutants also have unusual hypocotyl morphology in the dark, and may be deficient in some aspect of cell elongation (see below).

The bot1-5 mutant has morphological characteristics suggestive of a general deficiency in cell enlargement. Cotyledons, leaves, and flower parts were all foreshortened, and the mutant produced very few seed (Figure 1; data not shown). By complementation analyses, we determined that bot1-5 is allelic to the botero1-1 mutation (data not shown). This mutation confers similar phenotypes and maps to the same vicinity as botl-5 (H . H öft e, personal communication).

Finally, in contrast to the other mutants, the shy3, shy4, shy5, and shy6 mutants have no unusual morphological characteristics; instead, they exhibit phenotypes within the normal range of wild-type growth patterns. Muta-

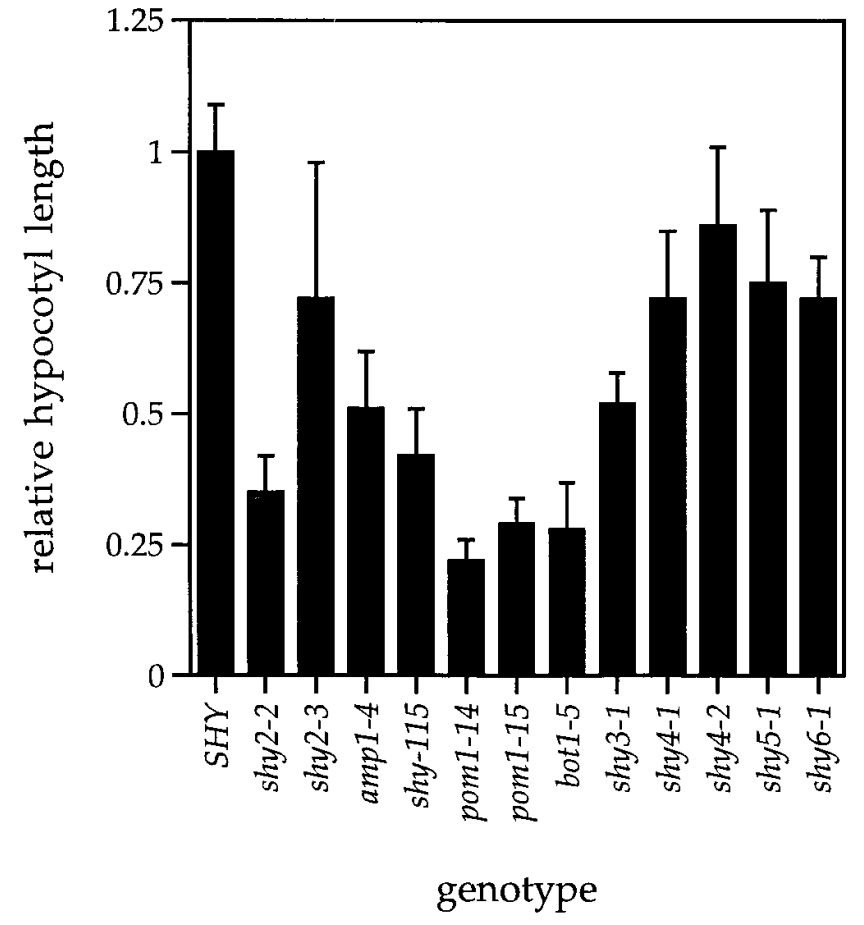

Figure 2.-Mean hypocotyl lengths of PH YB shy seedlings after 6 days of dark growth. Seedlings were grown on MS plates containing $2 \%$ sucrose, and hypocotyl lengths were normalized to the hypocotyl length of wild-type seedlings. Data from three experiments were pooled. Error bars indicate standard deviations. In the experiment in which shy2-3, bot1-5, and shy4-2 data were gathered, the wild-type measurement had a relative standard deviation of 2.5 times that shown. In each case, the shy mutant hypocotyl lengths were statistically significantly shorter than the wild-type hypocotyl length ( $P<0.05$ for shy4-2, $P<0.001$ for the rest).

tions at three loci, shy4, shy5, and shy6, are recessive, and one mutation, shy3-1, is semidominant (Table 2). As described below, shy3-1 and shy5-1 mutants have quantitative phenotypesthat suggest that they mayaffect PHYB signaling.

Dark phenotypes: Mutations that activate light-response pathways constitutively might be expected to cause phenotypes in the dark. In fact, a number of genes thought to play important rolesin photomorphogenesis were identified in screens for mutants that make leaves in the dark (see above). After 6 days in the dark, wildtype seedlings had a long hypocotyl, an unopened apical hook, small unexpanded cotyledons, and no leaf primordia ( Figures 2 and 3) . After 23 days, the apical hooks had opened, but the cotyledons were unexpanded and very few seedlings had visible leaf primordia (Figure 4, Table 6). We grew shy seedlings in the dark, and found that after 6 days all of them had significantly shorter hypocotylsthan the wild-type seedlings ( Figure 2). Some of the shy mutants also had open cotyled ons or leaf development, morphological characteristics that are normally limited to light-grown plants (Table 6, Figure 4).

The shyz-2 and brassinosteroid-deficient shy- 802 seed- 


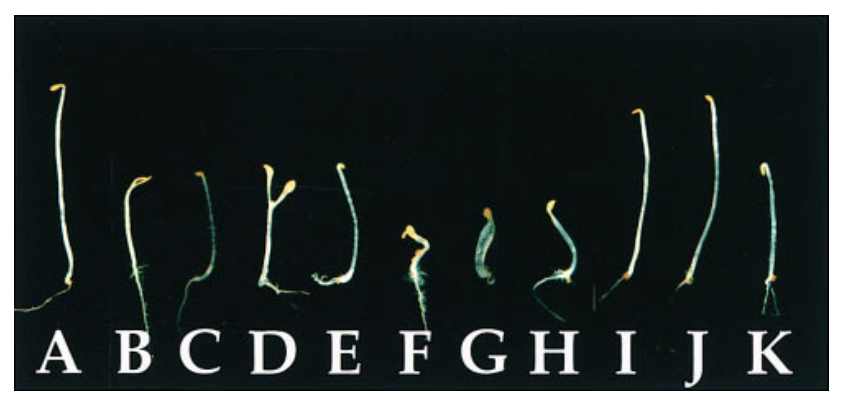

Figure 3.-PHYB shy seedlings after 6 days of dark growth. Seedlingswere grown on MS/ sucrose plates. Genotypes shown are (A) Landsberg erecta, (B) shy2-2, (C) shy2-3, (D) amp1-4, (E) shy-115, (F) pom1-15, (G) bot1-5, (H) shy3-1, (I) shy4-1, (J) shy5-1, and (K) shy6-1. shy4-1 and shy4-2 seedlings appeared similar to each other, and pom1-14 and pom1-15 seedlings appeared similar to each other. Therefore, only one mutant at each locus is shown.

lings resembled light-grown seedlings most closely, in that after 23 days they had structures resembling true leaves. Petioles were very short, and trichomes were visible (Figure 4). In this respect, these two mutants resembled the de-etiolated mutants, det1, det2, and cop1, described previously (Chory et al. 1989; Deng et al. 1991). Mutants carrying the weaker allele of SHY2, shy2-3, usually had expanded cotyledons after 23 days, but no visible leaf primordia (Figure 4, Table 6). The weaker brassinosteroid-deficient mutant shy-115 appeared similar to the wild type in the dark.

Several mutants had phenotypes that superficially resembled a de-etiolated phenotype, but diverged from a normal light growth pattern. amp1-4 seedlings had the most extreme phenotype after six days, having an open apical hook, expanded cotyledons, and appearance of leaf primordia. H owever, after 23 days, the organs in the positions where leaves would normally arise resembled elongated petioles or stemsmore than leaves, and yellow leaf blade material was absent or extremely abbreviated (Figure 4, Table 6). Similarly, the shy4-1, pom1-14, pom115, and bot1-5 mutants frequently developed branches resembling petioles (Figure 4, Table 6 ). This curious phenotype may represent a partial commitment to leaf development in these mutants. Alternatively, enhanced petiole or stem development may arise in conditions where the hypocotyl would otherwise elongate but is prevented from doing so by some physiological limitation caused by a shy mutation.

As well as making numerous petiole-like structures, the pom1-14 and poml-15 mutants had a unique dark hypocotyl morphology. The hypocotyls were quite crooked (Figures 3 and 4), and upon closer inspection, they appeared somewhat disorganized, with a rough surface (data not shown).

The remaining mutants (shy3-1, shy4-2, shy5-1, and shy6-1) had short hypocotyls, but otherwise looked similar to wild-type in the dark. shy3-1 seedlings had a slightly
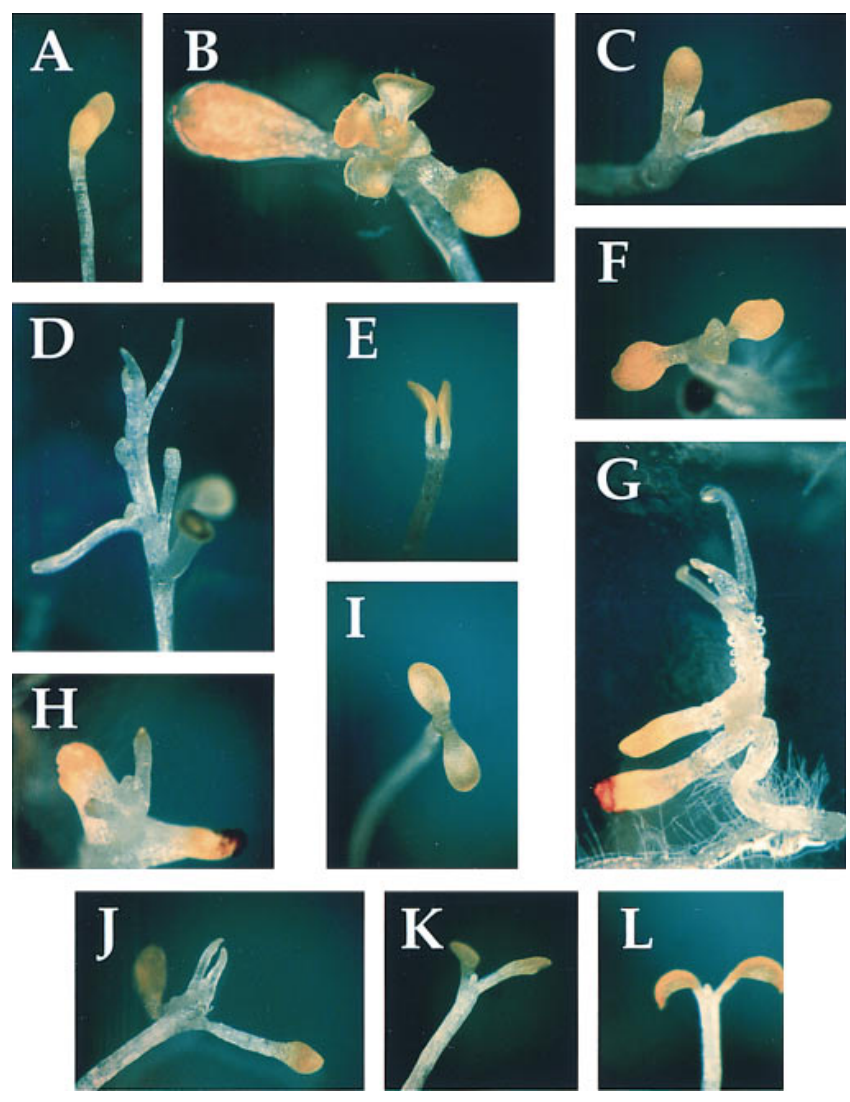

Figur e 4.-PH YB shy seedlings after 23 days of dark growth. Seedlingswere grown on MS/ sucrose plates. Genotypes shown are (A) Landsberg erecta, (B) shy2-2, (C) shy2-3, (D) amp1-4, (E) shy-115, (F) shy-802, (G) pom1-15, (H) bot1-5, (I) shy3-1, ( J) shy4-1, (K) shy5-1, and ( L) shy6-1. shy4-1 and shy4-2 seedlings appeared similar to each other, and pom1-14 and pom1-15 seedlings appeared similar to each other. Therefore, only one mutant at each locus is shown.

open apical hook after 6 days. All had open apical hooks after 23 days, but the cotyledons were unexpanded and few seedlings had leaves (Figure 4, Table 6). Each of the mutants had a slightly higher incidence of leaf primordium formation than the wild type (Table 6). We do not know the reason for this quantitative difference, but it may conceivably be an indirect consequence of having shorter hypocotyls.

$H$ ypocotyl elongation responses to red, blue, and farred light: If a SH Y protein activity is normally modulated by a light-signaling pathway, then mutation of the gene encoding that protein might cause the pathway to be more constitutive and less regulatable. In such a case, PH YB shy mutant seedlings would have a decreased response to light because of their "partially responding" baseline state, and they should be less sensitive to additional red light than wild-type seedlings. To examine this possibility, we tested the fluence rate-response behavior of the PH YB shy plantsfor hypocotyl el ongation in constant red light. Figure 5, A and B, show the hypocotyl lengths of 6-day-old shy mutant seedlings grown under 
TABLE 6

Leaf formation by shy mutants after 23 days in the dark

\begin{tabular}{lccc}
\hline shy genotype & Zero leaves & $\begin{array}{c}\text { Two or } \\
\text { more leaves }^{\mathrm{a}}\end{array}$ & $\begin{array}{c}\text { Fraction with } \\
\text { leaves }^{\mathrm{a}}\end{array}$ \\
\hline SHY & 68 & 9 & 0.12 \\
shy2-2 & 3 & $58^{\mathrm{c}}$ & 0.95 \\
shy2-3 & 39 & 19 & 0.33 \\
amp1-4 & 0 & $55^{\mathrm{b}}$ & 1.00 \\
shy-115 & 16 & 13 & 0.45 \\
shy-802 & 0 & $22^{\mathrm{c}}$ & 1.00 \\
pom1-14 & 0 & $44^{\mathrm{b}}$ & 1.00 \\
pom1-15 & 0 & $25^{\mathrm{b}}$ & 1.00 \\
bot1-5 & 0 & $25^{\mathrm{b}}$ & 1.00 \\
shy3-1 & 18 & 9 & 0.33 \\
shy4-1 & 2 & $17^{\mathrm{b}}$ & 0.89 \\
shy4-2 & 18 & 0 & 0 \\
shy5-1 & 28 & 8 & 0.22 \\
shy6-1 & 17 & 5 & 0.23 \\
\hline
\end{tabular}

a Number of seedlings with leaves or other lateral organs. Branches, leaves, petioles, and undifferentiated primordia arising from the apex were all counted.

${ }^{b}$ Leaves resembled elongated stems or petioles with small or absent leaf blades (Figure 4).

'Leaves resembled true leaves (Figure 4).

different fluence rates, normalized in each case to the hypocotyl length of the same strain in the dark. Most of the shy mutants responded to the same range of fluence rates as the wild type did, having hypocotyl lengths of $\sim 30 \%$ of their hypocotyl lengths in the dark at the highest fluence rate tested (Figure 5, A and B). As expected, the phyB-1 mutant was less sensitive to red light, showing a minimum hypocotyl length of $\sim 80 \%$ of its dark hypocotyl length.

The shy2-2, pom1-14, and pom1-15 mutants showed altered hypocotyl responses to red light. shy2-2 had a decreased response at the highest fluence rate tested (40-45 $\mu \mathrm{mol} \cdot \mathrm{m}^{-2} \cdot \mathrm{sec}^{-1}$ ), with a minimum hypocotyl length of $\sim 60 \%$ of its dark hypocotyl length (Figure 5A). In contrast, the pom1-14 and pom1-15 mutants had longer hypocotyls at very low fluence rates $(<0.1 \mu \mathrm{mol}$. $\mathrm{m}^{-2} \cdot \mathrm{sec}^{-1}$ ) than in the dark (Figure 5A). They thus showed the opposite response from the wild type in this fluence range. At higher fluence rates $\left(>1 \mu \mathrm{mol} \cdot \mathrm{m}^{-2}\right.$ - $\sec ^{-1}$ ), they had a normal response to red light, reaching a minimum hypocotyl length of $\sim 70 \%$ of their dark hypocotyl length (Figure 5A). Because the shy2-2, pom114, and pom1-15 mutants are each extremely short in the dark (Figures 2 and 3, data not shown), these observations may reflect an inherent limitation in the degree to which red light can inhibit hypocotyl elongation. H owever, the bot1-5 mutant, responded proportionately similarly to the wild type despite having a very short hypocotyl in the dark (Figures 2 and $5 \mathrm{~B}$ ). This observation suggests that the shy2-2, pom1-14, and pom1-15 mutants do not reach a minimum attainable hypocotyl length in these experiments, and that the mutations may thus affect red light signaling or response path ways.

To test whether the shy mutations may affect response pathways downstream of other photoreceptors, we also performed fluence rate response experiments in blue and far-red light. We found that all the mutants responded to both types of light with the same threshold and saturation characteristics as the wild type (Figure 5, C and D; data not shown). In particular, the shy2-2 and pom1-15 mutants had normal responses to both blue light (Figure 5C) and far-red light (Figure 5D). Therefore, the mutations have not affected a blue or far-red light signaling pathway.

Root elongation: Rather than repressing cell expansion in all seedling tissues, PH YB causes a redistribution of growth away from the hypocotyl and toward the roots and cotyledons. Thus, light-grown phyB mutant seedlings have a longer hypocotyl but a shorter root and smaller cotyledons than wild-type seedlings (Reed et al. 1993; N eff and Van Volkenburgh 1994). To determine whether the shy mutations affect elongation in multiple tissues, we measured the root lengthsof phyB-1 shy seedlings grown in red light. This assay was particularly useful for distinguishing between mutants affected in regulatory functions, which should have roots longer than the phyB-1 seedlings, and mutants affected specifically in elongation functions, which would be expected to have shorter roots than phyB-1 seedlings. Mutants with roots the same length as phyB-1 seedlings could belong to either group. As shown in Figure 6, wild-type (PHYB SH Y), amp1-4, shy2-2, and shy5-1 seedlings had significantly longer roots than phyB-1 seedlings, suggesting that these mutations may affect the redistribution of growth controlled by light. shy2-3, shy-802, pom114, pom1-15, bot1-5, shy4-2, and shy6-1 seedlings had significantly shorter roots than SHY seedlings, suggesting that these mutations affect functions specific to cell elongation. The remaining mutants (shy-115, shy31 , and shy4-1) had roots of similar length as the phyB parent strain.

Flowering time: In addition to having elongation phenotypes, phyB mutants flower early (Got o et al. 1991; Reed et al. 1993; Bagnal I etal. 1995). Plants that overexpress PHYB also flower early (Bagnall et al. 1995). We assessed flowering times in short days, under which conditions the difference in flowering time between wild-type and phyB plants is greatest. We measured both the time the plantstook to flower (DF, daysto flowering) and the number of leaves at the time of flowering ( $L N$, leaf number) in both PHYB and phyB-1 backgrounds. The results differed slightly from experiment to experiment, possibly because light conditions in our growth chamber varied (see mat er ial s and met hods). Therefore, we consider as meaningful only results where we observed a statistically significant difference between SHY and shy plants in the majority of experiments. We present data for a subset of the mutants in Figure 7 and 
A

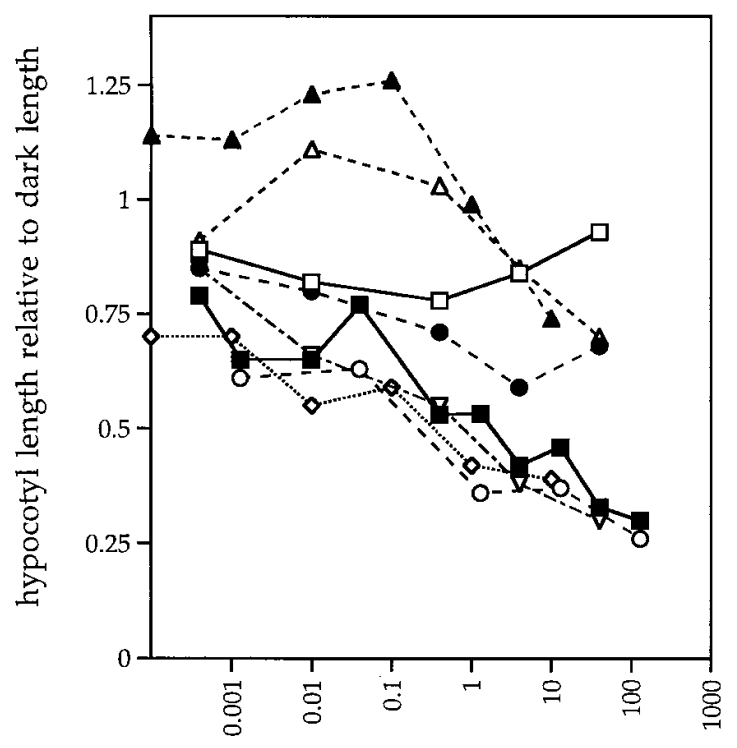

red light fluence rate, $\mu$ moles $/ \mathrm{m}^{2} \mathrm{sec}$
B

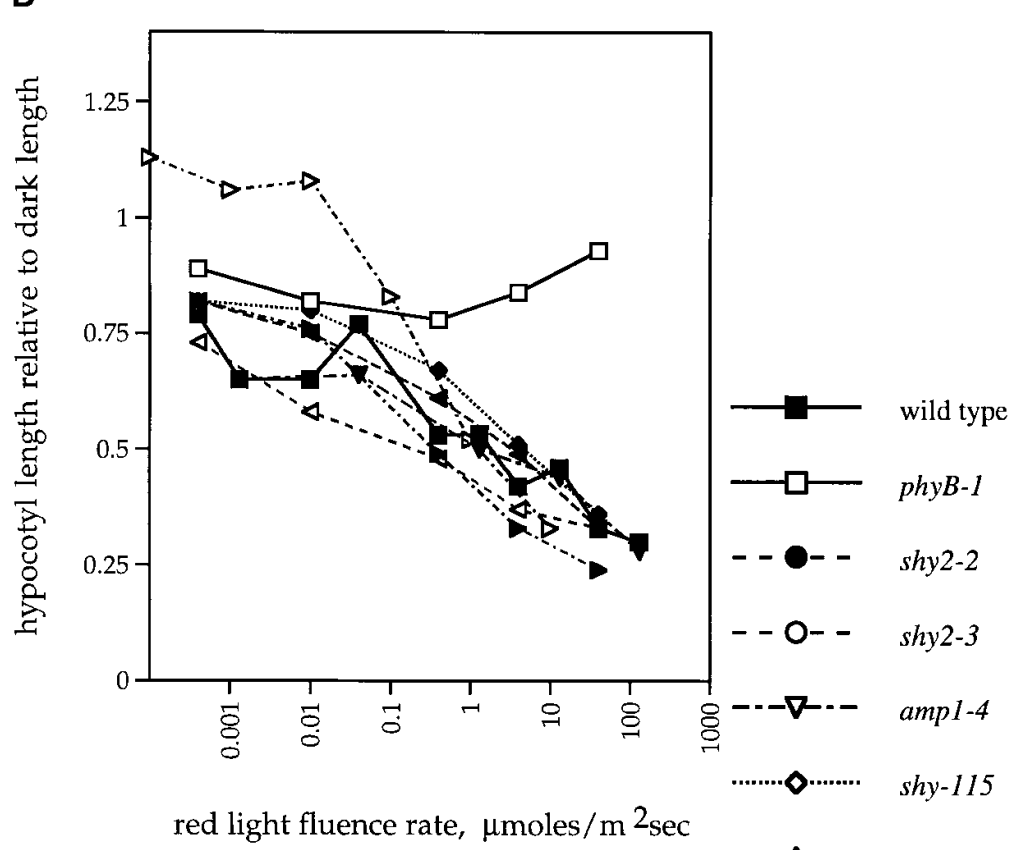

- - - - - poml-14

$---\Delta---\quad$ poml-15

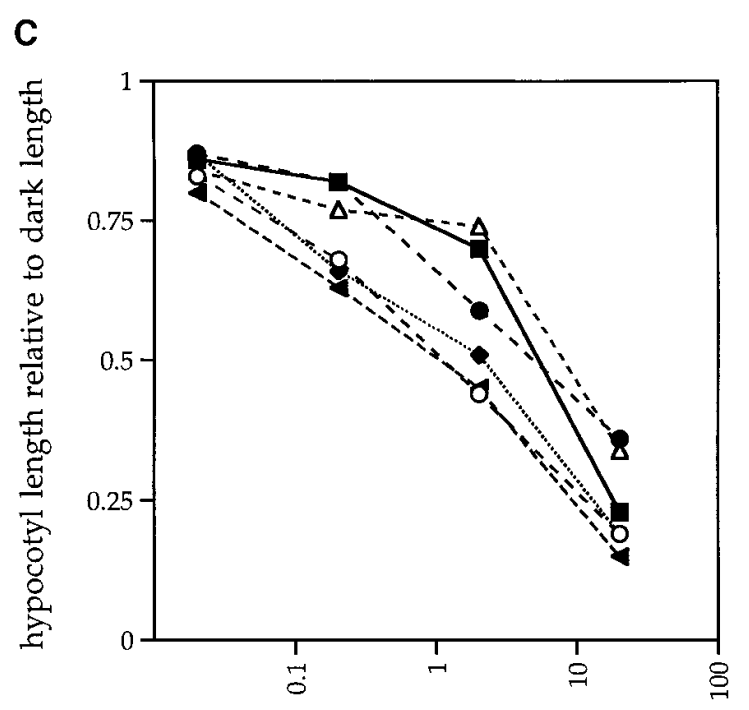

blue light fluence rate, $\mu$ moles $/ \mathrm{m}^{2} \mathrm{sec}$

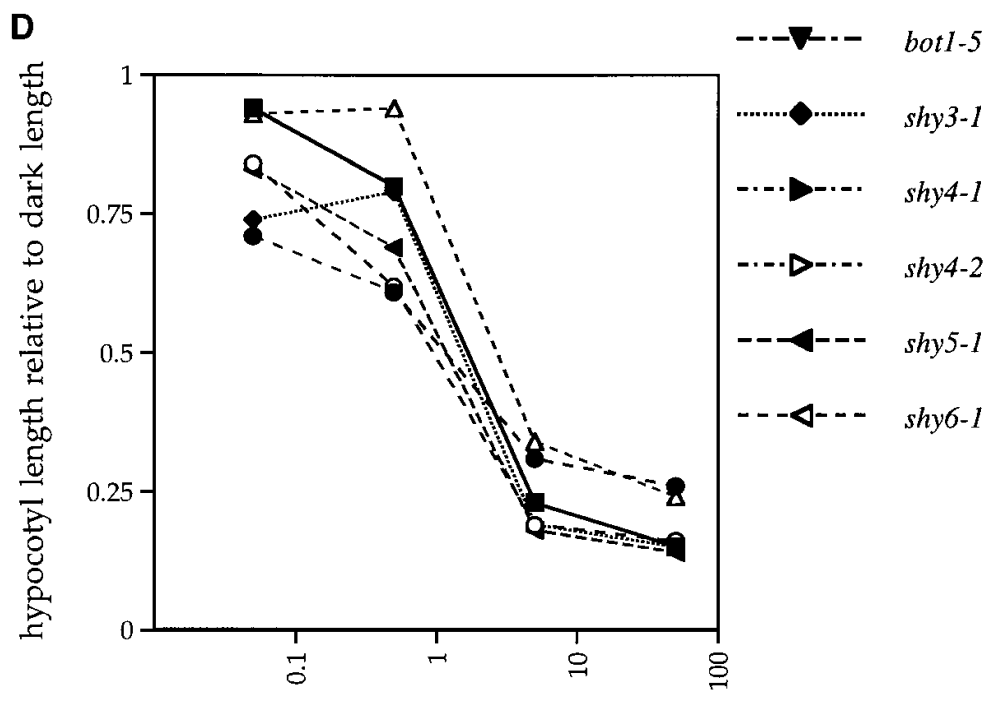

far red light fluence rate, $\mu$ moles $/ \mathrm{m}^{2} \mathrm{sec}$

Figur e 5.-H ypocotyl length response of shy seedlings to different fluence rates of red, blue, or far-red light. For each curve, data are normalized to the hypocotyl length of the same genotype in the dark. ( $A$ and B) Response to red light. Curves are split into two graphs for clarity, and data for wild type and phyB-1 are shown in both graphs. Each data point is the mean hypocotyl length of 20-40 seedlings, and standard deviations were generally $10-20 \%$ of the mean. Repetitions of this experiment gave similar results (data not shown). (C and D) Response to blue light (C) and far-red light (D). Shown are shy2-2, shy2-3, pom1-15, shy3-1, and shy5-1 mutants. amp1-4, shy-115, bot1-5, shy4-1, shy4-2, and shy6-1 mutants all responded similarly to wild type in both blue and far-red light (data not shown).

summarize our consensus results from six experiments in Table 7. Four of the mutations affected flowering time significantly.

One shy mutation, shy5-1, delayed flowering in both PHYB and phyB-1 backgrounds (Figure 7, Table 7). In the PHYB background, shy5-1 also caused more leaves to form before flowering, whereas in the phyB-1 background, the leaf number was normal. These data suggest that the SH Y 5 gene product may normally antagonize the activity of PHYB. 


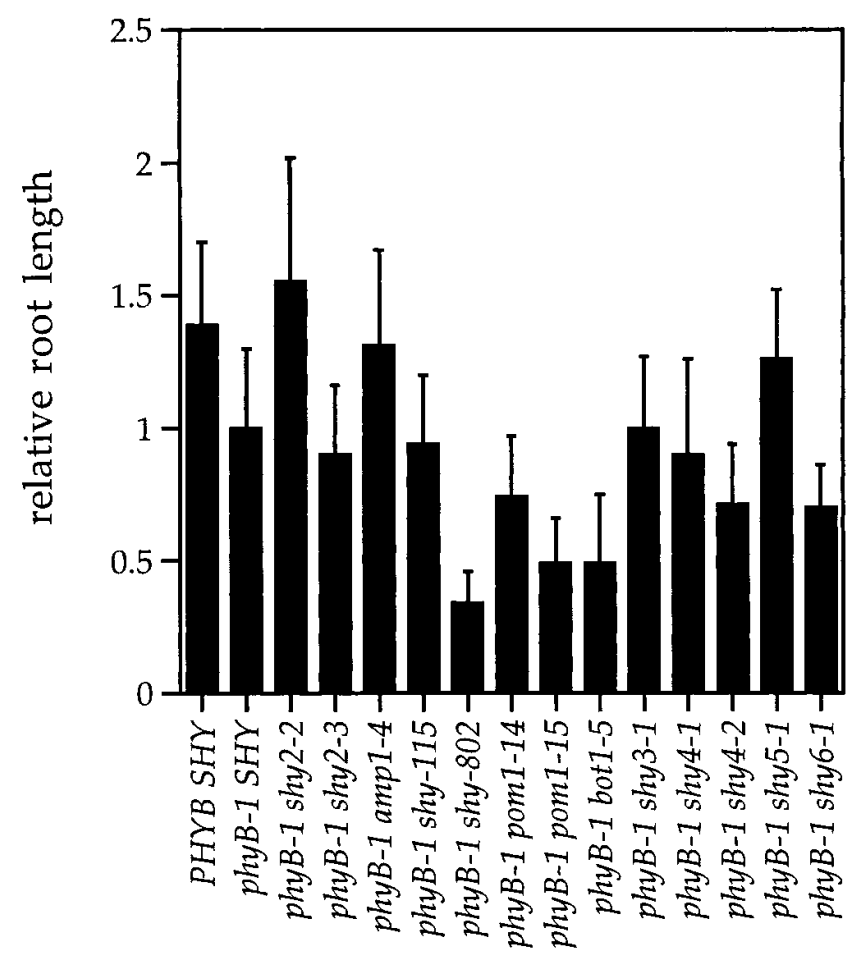

\section{genotype}

Figure 6.-Root lengths of phyB-1 shy seedlingsin red light. shy seedling root lengthswere normalized to the root length of phyB-1 seedlings. Data from four experiments were combined. Error barsindicate standard deviations of measurements. Normalized root lengths were tested for significant difference from phyB-1 root length by t-test. All genotypes except phyB-1 shy-115, phyB-1 shy3-1, and phyB-1 shy4-1 showed a difference from phyB-1 root lengths at $95 \%$ confidence or more. $P$ values from t-tests for comparison with phyB-1 root lengths were wild type, $\mathrm{P}<0.001$; phyB-1 shy2-2, $\mathrm{P}<0.001$; phyB-1 shyz-3, $\mathrm{P}<$ 0.05; phyB-1 amp1-4, $\mathrm{P}<0.001$; phyB-1 shy-115, $\mathrm{P}<0.5$; phyB-1 shy-802, $\mathrm{P}<0.001$; phyB-1 pom1-14, $\mathrm{P}<0.025$; phyB-1 pom1$15, \mathrm{P}<0.001$; phyB-1 bot1-5, $\mathrm{P}<0.001$; phyB-1 shy3-1, $\mathrm{P}=1$; phyB-1 shy4-1, $\mathrm{P}<0.1$; phyB -1 shy4 $-2, \mathrm{P}<0.001$; phyB-1 shy5-1, $P<0.001 ;$ and phyB-1 shy6-1, $P<0.001$.

The shy2-2 and shy3-1 mutations caused early flowering in the PHYB background, but had no effect on flowering time in the phyB-1 background. Both PHYB shy2-2 and PH YB shy3-1 mutant plants also flowered with fewer leaves than wild-type plants. Although they flowered at the normal time, phyB-1 shy3-1 plants had extra leaves. The early flowering of shy2-2 and shy3-1 mutants in the PHYB background suggests that $\mathrm{SH} Y 2$ and $\mathrm{SH} Y 3$ have significant regulatory functions.

As mentioned above, amp1-4 plants made leaves more quickly than AM P plants. This accelerated leaf production was accompanied by an acceleration of flowering in both PHYB and phyB-1 backgrounds. However, the effect of the amp1-4 mutation on the number of leaves made before flowering correlated poorly with its effect on flowering time. Thus, in some experiments, the leaf number was significantly greater than for the corresponding SH Y plants, and in other experiments, the leaf number was significantly smaller (data not shown). This observation suggests that the accelerated flowering of amp1-4 plants may be a secondary consequence of its rapid leaf production.

The remaining shy mutations ( shy2-3, shy-115, pom114, pom1-15, bot1-5, shy4-1, shy4-2, and shy6-1) had no effect on the number of days to flowering in either PH YB or phyB-1 backgrounds (Table 7). Three of these, shy2-3, bot1-5, and shy6-1, affected leaf number in one genetic background (Table 7). (The shy2-3 mutation caused extra leaf formation in the phyB-1 background, the bot1-5 mutation caused flowering with fewer leaves in the PH YB background, and the shy6-1 mutation caused extra leaf formation in the PH YB background.)

\section{DISCUSSION}

The phyB-1 mutation creates a stop codon and is probably a null allele (Reed \& al. 1993). Therefore, the suppressor mutations described here most likely bypass the requirement for PH YB for inhibiting hypocotyl elongation. The shy mutations may identify downstream mediators of PH YB signaling, regulators of other environmental response pathways, or biochemical or metabolic functions needed for hypocotyl elongation. The occurrence of single mutations at several of the loci indicates that we have not saturated the screen. Additional known loci that can mutate to give a short hypocotyl phenotype include the AXR genes and the DET / COP/FUS genes (Fankhauser and Chory 1997).

Based on the mutant phenotypes, the best candidates for genes that regulate light responses are SH Y 2, SH Y 3 , and SH Y 5. The recessive shy5-1 mutation suppresses all of the phyB-1 phenotypes we tested. phyB-1 shy5-1 plants have a shorter hypocotyl than the starting phyB-1 mutant, they have a longer root, and they flower late. For each phenotype, the suppression is partial in that the phyB-1 shy5-1 plants still differ from wild-type plants. This may indicate that the mutation is a partial loss-offunction allele or that the mutated function is encoded by more than one gene. Taken together, the results suggest that the SH Y 5 gene may encode a function that opposes the activity of $\mathrm{PH} Y \mathrm{YB}$, acting either downstream of PHYB in a light regulatory pathway or in a separate branch of the control network.

The shy2 mutants have several striking phenotypes that suggest that SHY2 may be an important mediator of red light responses. shy2-2 plants make leaves in the dark, respond only slightly to red light for control of hypocotyl elongation, have an elongated root in the phyB-1 background, and flower early in the PH YB background. The weaker shy2-3 mutation caused less profound effects on development than shyz-2, e.g., expanded cotyledons without obvious leaf formation in the dark. Like the shyz-2 and shy2-3 mutations described 
$A$
$A$
0
0
0
0
0
0
0
0
0
0
0
0
0
0
0
0
0
0
0
0
0
0
0
0

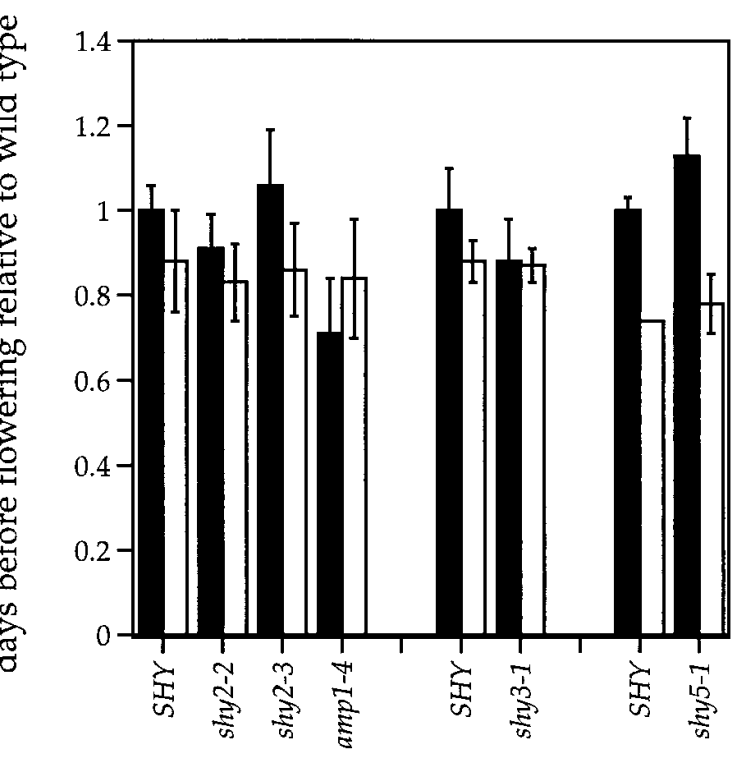

SHY genotype

B

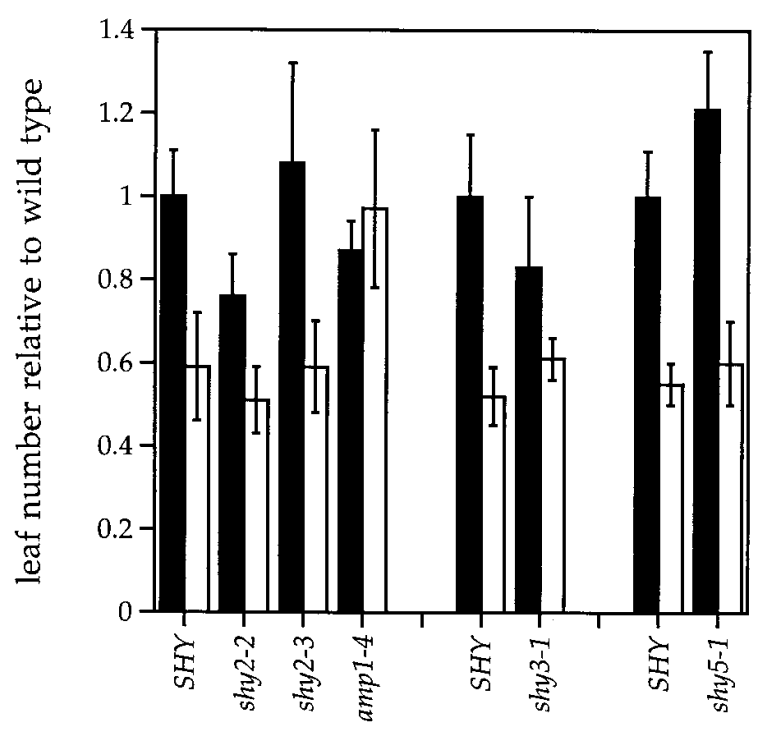

SHY genotype

PHYB background

phyB-1 background

Figure 7.-Flowering of selected PHY shy and phyB-1 shy plants in short days. (A) days to flower; (B) leaf number at time of flowering. Error bars indicate standard deviations. Shown are data for selected genotypes from three separate experiments. To facilitate comparison between different experiments, data have been normalized to the wild-type flowering time and $L N$ in each case. In the experiments shown, the flowering times ( $A$ ) for PH YB shy2-2, PH YB amp1-4, PH YB shy3-1, PH Y B shy5-1, and phyB-1 shy5-1 were significantly different from the corresponding $\mathrm{SH} Y$ plants $(P<0.05$ or less) ;- here, the previously described shy2-1 mutation is semidominant, causes cotyledon expansion in the dark, and causes upcurled leaves in the light (Kim et al. 1996). The semidominance of all three shy2 alleles is consistent with the mutations being hypomorphic, hypermorphic, or neomorphic. The frequency with which we have obtained shy2 alleles might indicate that the mutations are hypomorphic (decreased function), in which case SH Y2 may normally repress phytochrome-mediated development in the dark. If the mutations are hypermorphic, SHY2 may normally activate de-etiolation in response to light. If the mutations are neomorphic, they may reveal otherwise cryptic effects of some other regulatory pathway on seedling development.

Like shy2 mutants, mutants of the det/ cop/ fus class make leaves in the dark. However, the morphological phenotypes of shy2 mutants are quite distinct from the phenotypes of mutants of the det/cop/ fus class, such as det1-1. For example, det1-1 mutant seedlings do not have curled leaves characteristic of shy2 seedlings, and shy2 mutant seedlings do not overproduce anthocyanin as detl seedlings do. Thus, SH Y2 probably regulates development in a manner different from the DET/COP/ FUS gene products.

The last of the mutations with a substantial effect on both flowering and hypocotyl elongation is shy3-1. Interestingly, shy3-1 partially suppressed the flowering phenotype caused by a phyB-1 mutation, but in a PH YB background, it caused early flowering, as phyB-1 does. Thus, PH YB shy3-1 plants flowered with reduced leaf number compared to wild-type plants, whereas phyB-1 shy3-1 plants flowered with increased leaf number compared to phyB-1 plants. This dampening of the effect of PHYB on leaf number may indicate that SH Y3 interacts with PHYB to control flowering (but see below). The semidominance of the shy3-1 mutation for hypocotyl length phenotypes is consistent with either a gain-offunction or loss-of-function type of allele, so the normal role of $\mathrm{SH} Y 3$ in development could be either to transmit the PHYB signal or to antagonize it.

At first blush, the early flowering conferred by the shyz-2 and shy3-1 mutations would seem counter to the expectation (fulfilled by shy $5-1$ ) that a regulatory suppressor mutation should affect any phenotypes in the opposite sense as the starting phyB-1 mutation. H owever, interpretation of the flowering phenotypes is complicated by the finding that when overexpressed, PHYB causes early flowering (Bagnall et al. 1995). This suggests either that overactivation of $\mathrm{PHYB}$ response pathways causes early (rather than late) flowering or that overexpressed PHYB can co-opt another pathway that

and the leaf numbers, (B) for PHYB shy2-2, phyB-1 shy2-2, PHYB amp1-4, phyB-1 amp1-4, PHYB shy3-1, phyB-1 shy3-1, and PH YB shy5-1 were significantly different from the corresponding SH Y plants ( $P<0.05$ or less). Flowering data from several experiments are summarized in Table 7. 
TABLE 7

Summary of developmental phenotypes of shy mutants ${ }^{\mathrm{a}}$

\begin{tabular}{|c|c|c|c|c|c|c|c|}
\hline \multirow[b]{3}{*}{ shy genotype } & \multirow[b]{3}{*}{ Dark development } & \multirow[b]{3}{*}{$\mathrm{RL}$ response } & \multirow[b]{3}{*}{ Root length } & \multicolumn{4}{|c|}{ Flowering ${ }^{c}$} \\
\hline & & & & \multicolumn{2}{|c|}{ PHYB background } & \multicolumn{2}{|c|}{ phyB-1 background } \\
\hline & & & & DF & LN & DF & LN \\
\hline $\mathrm{SHY}$ & Long hyp., small cotyledons & + & Medium & Normal & Normal & Normal & Normal \\
\hline shy2-2 & Leaves & $+1-$ & Long & Early & Low & Normal & Normal \\
\hline shy2-3 & Expanded cotyledons & + & Short & Normal & Normal & Normal & High \\
\hline ampl-4 & Stem-like organs & + & Long & Early & Low & Normal & High \\
\hline shy-115 & Normal & + & Normal & Normal & Normal & Normal & Normal \\
\hline shy-802 & Leaves & & Short & & & & \\
\hline pom1-14 & Twisted hyp.; stem-like organs & $+1-\mathrm{b}$ & Short & Normal & Normal & Normal & Normal \\
\hline pom1-15 & Twised hyp.; stem-like organs & $+1-b$ & Short & Normal & Normal & Normal & Normal \\
\hline bot1 -5 & Stem-like organs & + & Short & Normal & Low & Normal & Normal \\
\hline shy3-1 & Normal & + & Normal & Early & Low & Normal & High \\
\hline shy4-1 & Stem-like organs & + & Normal & Normal & Normal & Normal & Normal \\
\hline shy4-2 & Normal & + & Short & Normal & Normal & Normal & Normal \\
\hline shy5-1 & Normal & + & Long & Late & High & Late & Normal \\
\hline shy6-1 & Normal & + & Short & Normal & High & Normal & Normal \\
\hline
\end{tabular}

a Phenotypes of shy mutants are described relative to the corresponding SHY strain.

${ }^{b}$ pom1-14 and pom1-15 seedlings have an altered response to low fluence rates (Figure 5).

' Shown are the consensus results for days to flower (DF) and leaf number (LN) at time of flowering. In each column, the phenotype of the SH Y plants is taken as normal. Thus, although phyB-1 SH Y plants flower earlier than PH YB SH Y plants, both are considered "normal" for the purpose of comparing their phenotypes to those of the corresponding shy plants. hyp., hypocotyl.

normally promotes flowering. For example, phytochrome A normally inhibits elongation and also promotes flowering ( $\mathrm{N}$ ag at an i et al. 1993; Par ks and Q uail 1993; Wh it el am et al. 1993; Johnson et al. 1994; Reed et al. 1994). In either case, a shy mutation might cause early flowering by acting similarly to overexpressed PHYB. Another possible explanation for why these shy mutations cause early flowering could be that they affect flowering time indirectly through effects on elongation. phyB-1 plants make leaves at a slower rate than wild-type plants, perhaps as a result of extra elongation growth at the expense of new organ formation (Koornneef et al. 1995; data not shown). If flowering depends in part on formation of a threshold number of leaves, then the slower rate of leaf formation in phyB-1 plants may actually delay flowering and partially compensate for the propensity of such plants to flower with fewer leaves. In that case, a mutation that suppressed the elongation phenotypes of a phyB-1 mutant would cause earlier flowering, as the shy2-2 and shy3-1 mutations do.

Our finding of an allele of AMP (amp1-4) and presumed brassinosteroid auxotrophs (shy-115 and shy-802) in this screen underscores the probable relevance of plant hormone signaling path ways to seedling de-etiolation. The amp mutant overproduces cytokinin ( $\mathrm{Ch}$ a ud hury et al. 1993; Chin-At kins et al. 1996), and applications of cytokinin to dark-grown seedlings can induce aspects of a de-etiolated phenotype (Ch or y et al. 1994; Ch in-A t kins et al. 1996). H owever, dark-grown amp mutant seedlings make organs resembling stems or petioles rather than leaves, and the altered phyllotaxy of amp mutants cannot be mimicked by altering light conditions. These observations suggest that the connection of AMP to photomorphogenesis may be indirect. Brassinosteroids have been implicated in repressing seedling de-etiolation because mutants isolated as having partially de-etiolated phenotypes in the dark have turned out to be brassinosteroid auxotrophs ( $\mathrm{Li}$ \& al. 1996; Szeker es et al. 1996). Like shy-802, mutants such as det2-1 make leaves in the dark, although the plastids do not differentiateas they do in other de-etiolated mutants such as detl (Ch ory et al. 1991). It remains to be determined whether these observations reflect direct regulation of brassinosteroid physiology by light. Other workers have reported evidence suggesting that auxin and light signaling are connected. For example, Nicotiana plumbaginifolia phytochrome mutants have been found to have increased auxin levels ( $\mathrm{Kr}$ aepiel et al. 1995), and some auxin-resistant mutants are short and have partiallyde-etiolated phenotypesin the dark ( $\mathrm{L}$ in col net al. 1990; T impt e et al. 1992; Leyser et al. 1996; Cernac et al. 1997; A. Sonawal a and J. W. Reed, unpublished observations). In this regard, it is interesting that shy2 mutants share some phenotypes, such as curled leaves, with axr3 mutants (Leyser et al. 1996).

The remaining shy mutations suppress only the long hypocotyl phenotype of the starting phyB-1 mutant, but do not suppress the root elongation or flowering time phenotypes. The pom1-14, pom1-15, bot1-5, shy4-2, and shy6-1 mutations actually cause the root to be shorter 
than that of phyB-1 plants. Thisobservation suggests that either these mutations primarily affect a cell elongation or enlargement function, or they affect cell division rates in the root. The morphological variety among these mutants suggests that the different mutations may affect distinct aspects of growth. Those with quantitative effects but having otherwise normal shape (e.g., shy4-2 or shy6-1) may affect a control function. Those conferring aberrant morphology (e.g., pom1-14 or bot1-5) may affect part of the cellular machinery that elongates cells, synthesizes cell walls, or determines cell polarity.

The twisted hypocotyls of dark-grown pom1 mutants resemble those of dark-grown procustel mutants (Desnos et al. 1996), suggesting that POM 1 and PR OCUSTE 1 have related functions. Apparently, these genes are required for proper hypocotyl elongation in the dark. Whereas the procustel mutants show a biphasic hypocotyl elongation curve in blue light, however, our poml mutants have a normal blue light response but an aberrantresponse in red light. POM 1 is also required for elongation and control of cortical cell enlargement in roots ( $\mathrm{H}$ auser et al. 1995) and for proper epidermal cell differentiation (Schneider \& al. 1997). POM1 therefore plays a role in growth and/ or shape determination of cells of various organs.

None of the putative regulatory mutations we have identified (shy2-2, shy2-3, shy3-1, or shy5-1) is epistatic to thephyB-1 mutation for the hypocotyl length phenotype. This indicates that these mutations either cause incomplete defects in the corresponding genes or affect redundant functions. The results reinforce our view of light signal transduction as a network of interacting components rather than as a collection of linear pathways (Reed and Chory 1994). As well, light signaling is surely intimately coupled to more general developmental control mechanisms. Further analysis of the shy mutants reported here may contribute to unraveling the complex regulatory pathways that mediate control of plant development by a variety of environmental factors.

We thank P. Nagpal and A. Pepper for helpful discussions; A. Chin-At kins, M.-T. H auser, H. H öft e, H.-G. Nam, and T. Wada for sending seeds; S. Wh it fi el $d$ for help with color figures; and an anonymous reviewer for a careful reading of the manuscript. This work was supported by National Institutes of $\mathrm{H}$ ealth $(\mathrm{NIH})$ grant R29GM 52456 to J.W.R. Early stages of this work were supported by grants from the National Science Foundation and the International H uman Frontier Science Program to J.C., and bya U. S. Department of EnergyEnergy Biosciences postdoctoral fellowship from the Life Sciences Research Foundation (Baltimore, MD) to J.W.R. J.C. is currently supported by NIH grant R01-GM 52413 and is an associate investigator of the Howard Hughes Medical Institute.

\section{LITERATURE CITED}

Ahmad, M., and A. R. Cashmore, 1993 HY4 gene of A. thaliana encodes a protein with characteristics of a blue-light photoreceptor. Nature 366: 162-166.

Ah mad, M., and A.R. Cash mor e, 1996 The pef mutants of A rabidopsis thaliana define lesions early in the phytochrome signaling pathway. Plant J. 10: 1103-1110.

Ahmad, M., and A. R. Cashmore, 1997 The blue-light receptor cryptochrome 1 shows functional dependence on phytochrome A or phytochrome B in Arabidopsis thaliana. Plant J. 11: 421-427.

Ang, L.-H., and X.-W. Deng, 1994 Regulatory hierarchy of photomorphogenic loci: genetic evidence for direct interaction between COP1 and HY 5 in controlling Arabidopsis seedling development. Plant Cell 6: 613-628.

Aukerman, M. J., M. H irsch fel d, L. West er, M. Weaver, T. Cl ack et al., 1997 A deletion in the PHYD gene of the Arabidopsis Wassilewskija ecotype defines a role for phytochrome $D$ in red/ far-red light sensing. Plant Cell 9: 1317-1326.

Bagnall, D. J., R. W. King, G. C. Whit el am, M. T. Boyl an, D. Wagner et al., 1995 Flowering responses to altered expression of phytochrome in mutants and transgenic lines of Arabidopsis thaliana (L.) Heynh. Plant Physiol. 108: 1495-1503.

Barnes, S. A., R. B. Quaggio, G. C. Wh it el am and N.-H . Chua, 1996 fhyl definesa branch point in phytochrome $A$ signal transduction pathways for gene expression. Plant J. 10: 1155-1161.

Bell, C., and J. Ecker, 1994 Assignment of 30 microsatellite loci to the linkage map of Arabidopsis. Genomics 19: 137-144.

Bot t 0, J. F., R. A. Sánchez, G. C. Whit el am and J. J. Casal, 1996 Phytochrome $A$ mediates the promotion of seed germination by very low fluences of light and canopy shade light in Arabidopsis. Plant Physiol. 110: 439-444.

Bowler, C., H. Yamagat a, G. N euhaus and N.-H. Chua, 1994 Phytochrome signal transduction pathways are regulated by reciprocal control mechanisms. Genes Dev. 8: 2188-2202.

Cabrera y Poch, H.L., C. A. Pet 0 and J. Chory, 1993 A mutation in the Arabidopsis DET 3 gene uncouples photoregulated leaf development from gene expression and chloroplast biogenesis. Plant J. 4: 671-682.

Carol, P., J. Peng and N. P. H arber d, 1995 Isolation and preliminary characterization of gas1-1, a mutation causing partial suppression of the phenotype conferred by the gibberellin-insensitive (gai) mutation in Arabidopsis thaliana (L.) Heynh. Planta 197: 414-417.

Casal, J. J., and H. Boccal andr 0, 1995 Co-action between phytochrome B and HY4 in Arabidopsis thaliana. Planta 197: 213-218.

Cernac, A., C. Lincoln, D. Lammer and M. Estelle, 1997 The SAR 1 gene of Arabidopsis acts downstream of the AXR1 auxin response. Development 124: 1583-1591.

Ch amovit z, D. A., N. Wei, M. T. O st er I und, A. G. von Arnim, J. M. St aub et al., 1996 The COP9 complex, a novel multisubunit nuclear regulator involved in light control of a plant developmental switch. Cell 86: 115-121.

Chaudhury, A. M., S. Let ham, S. Craig and E. S. Dennis, 1993 ampl - a mutant with high cytokinin levels and altered embryonic pattern, faster vegetative growth, constitutive photomorphogenesis and precocious flowering. Plant J. 4: 907-916.

Chin-Atkins, A. N., S. Craig, C. H. Hocart, E. S. Dennis and A. M. Chaudhury, 1996 Increased endogenous cytokinin in the Arabidopsis ampl mutant corresponds with de-etiolation responses. Planta 198: 549-556.

Chory, J., 1992 A genetic model for light-regulated seedling development in Arabidopsis. Development 115: 337-354.

Chory, J., C. Pet 0, R. Feinbaum, L. Pratt and F. Ausubel, 1989 Arabidopsis thaliana mutant that develops as a light-grown plant in the absence of light. Cell 58: 991-999.

Chor $y, J$., and C. A. Pet 0, 1990 Mutations in the DET 1 gene affect cell-type-specific expression of light regulated genes and chloroplast development in Arabidopsis. Proc. Natl. Acad. Sci. USA 87: 8776-8780.

Chory, J., P. Nagpal and C. A. Pet 0, 1991 Phenotypic and genetic analysis of det2, a new mutant that affects light-regulated seedling development in Arabidopsis. Plant Cell 3: 445-459.

Chory, J., D. Reinecke, S. Sim, T. Wash burn and M. Brenner, 1994 A role for cytokinins in de-etiolation in A rabidopsis. Plant Physiol. 104: 339-347.

Clack, T., S. Matthews and R. A. Sharrock, 1994 The phytochrome apoprotein family in Arabidopsis is encoded by five genes: the sequences and expression of PHYD and PHYE. Plant Mol. Biol. 25: 413-428.

Clouse, S. D., M. Langford and T. C. M CMorris, 1996 A brassino- 
steroid-insensitive mutant in A rabidopsis thaliana exhibitsmultiple defects in growth and development. Plant Physiol. 111: 671-678.

Dehesh, K., C. Franci, B. M. Parks, K. A. Seel ey, T. W. Short et al., 1993 Arabidopsis H Y 8 locus encodes phytochrome A. Plant Cell 5: 1081-1088.

Deng, X.-W., T. Caspar and P. Q uail , 1991 cop1: a regulatorylocus involved in light-controlled development and gene expression in Arabidopsis. Genes Dev. 5: 1172-1182.

Desnos, T., V. O r bovíc, C. Bel I ini, J. Kronenber ger, M. Caboche et al., 1996 Procustel mutantsidentifytwo distinct genetic pathways controlling hypocotyl cell elongation, respectively in dark- and light-grown Arabidopsis seedlings. Development 122: 683-693.

Fankhauser, C., and J. Ch or y, 1997 Light control of plant development. Annu. Rev. Cell Dev. Biol. 13: 203-229.

Fel dmann, K. A., M. D. Marks, M. L. Christianson and R. S. Quat r ano, 1989 A dwarf mutant of A rabidopsis generated by T-DNA insertion mutagenesis. Science 243: 1351-1354.

Fur uya, M., 1993 Phytochromes: their molecular species, genefamilies, and functions. Annu. Rev. Plant Physiol. Plant Mol. Biol. 44: 617-645.

Goto, N., T. Kumagai and M. Koornneef, 1991 Flowering responses to light-breaks in photomorphogenic mutants of Arabidopsis thaliana, a long-day plant. Physiol. Plant 83: 209-215.

H auser, M.-T., A. Morikami and P. N. Benfey, 1995 Conditional root expansion mutants of Arabidopsis. Development 121: 12371252.

Hou, Y., A. G. von Arnim and X.-W. Deng, 1993 A new class of Arabidopsis constitutive photomorphogenic genes involved in regulating cotyledon development. Plant Cell 5: 329-339.

Johnson, E., M. Bradl ey, N. P. H arberd and G. C. Whit el am, 1994 Photoresponses of light-grown phyA mutants of A rabidopsis: phytochrome $A$ isrequired for the perception of daylength extensions. Plant Physiol. 105: 141-149.

Karim, F. D., H. C. Chang, M. Thier ren, D. A. Wassarman, T. Lavert y et al., 1996 A screen for genes that function downstream of Rasl during Drosophila eye development. Genetics 143: $315-329$

Kim, B. C., M. S. Soh, B. J. Kang, M. Furuya and H. G. Nam, 1996 T wo dominant photomorphogenic mutations of A rabidopsis thaliana identified assuppressor mutations of hy2. Plant J. 9: 441-456.

Konieczny, A., and F. Ausubel, 1993 A procedure for mapping Arabidopsis mutations using co-dominant ecotype-specific PCR based markers. Plant J. 4: 403-410.

Koornneef, M., E. Rolff and C.J.P. Spruit, 1980 Genetic control of light-inhibited hypocotyl elongation in A rabidopsis thaliana ( $L$.) H eynh. Z Pflanzenphysiol 100: 147-160.

Koornneef, M., M. Jorna, D. Brinkhorst-Van der Stran and C. Karssen, 1982 The isolation of abscisic acid (ABA) deficient mutants by selection of induced revertants in non-germinating gibberellin-sensitive lines of Arabidopsis thaliana (L.) Heynh. Theor. Appl. Genet. 61: 385-393.

Koornneef, M., C. Hanhart, P. Van Loenen-Martinet and H. B. De Vries, 1995 The effect of daylength on the transition to flowering in phytochrome-deficient, late-flowering and double mutants of Arabidopsis thaliana. Physiol. Plant. 95: 260-266.

Kraepiel, Y., K. Marrec, B. Sott a, M. Caboche and E. Miginiac, 1995 In vitro morphogenic characteristics of phytochrome mu tants in Nicotiana plumbaginifolia are modified and correlated to high indole-3-acetic acid levels. Planta 197: 142-146.

Leyser, H. M. O., F. B. Picket t, S. Dhar masiri and M. Est el I e, 1996 Mutations in the AXR3 gene of A rabidopsis result in altered auxin response including ectopic expression from the SAUR-AC1 promoter. Plant J. 10: 403-413.

Li, H.-M ., L. Alt schmied and J. Chory, 1994 Arabidopsis mutants define downstream branches in the phototransduction pathway. Genes Devel. 8: 339-349.

Li, J., P. Nagpal, V. Vit art, T. M cMorris and J. Chory, 1996 A role for brassinosteroids in light-dependent development of Arabidopsis. Science 272: 398-401.

Lin, Y., and C.-L. Ch eng, 1997 A chlorate-resistant mutant defective in the regulation of nitrate reductase gene expression in Arabidopsis defines a new H Y locus. Plant Cell 9: 21-35.

Lincoln, C., J. H. Britton and M. Estelle, 1990 Growth and development of the axr1 mutants of Arabidopsis. Plant Cell 2 1071-1080.

Mayer, R., D. Ravent os and N.-H . Chua, 1996 det1, cop1, and cop9 mutations cause inappropriate expression of several gene sets. Plant Cell 8: 1951-1959.

Misér a, S., A. J. Mull er, U. Weil and-H eidecker and G. Jürgens, 1994 The FUSCA genes of Arabidopsis: negative regulators of light responses. Mol. Gen. Genet. 244: 242-252.

Nagat ani, A., J. W. Reed and J. Chory, 1993 Isolation and initial characterization of A rabidopsis mutants that are deficient in phytochrome A. Plant Physiol. 102: 269-277.

Neff, M., and E. Van Vol kenburgh, 1994 Light-stimulated cotyledon expansion in A rabidopsis seedlings: The role of phytochrome B. Plant Physiol. 104: 1027-1032.

Neuhaus, G., C. Bowler, R. Kern and N.-H.Chua, 1993 Calcium/ calmodulin-dependent and -independent phytochrome signal transduction pathways. Cell 73: 937-952.

Niyogi, K., R. Last, G. Fink and B. Keit h, 1993 Suppressors of trpl fluorescenceidentify a newArabidopsisgene, TRP4, encoding the anthranilate synthase $\beta$ subunit. Plant Cell 5: 1011-1027.

0 yama, T., Y. Sh imura and K. O kada, 1997 The Arabidopsis HY5 gene encodes a bZIP protein that regulates stimulus-induced development of root and hypocotyl. Genes Dev. 11: 2983-2995.

Par ks, B. M., and P. H. Q uail , 1993 hy8, a new class of Arabidopsis long hypocotyl mutants deficient in functional phytochrome $A$ Plant Cell 5: 39-48.

Pepper, A., and J. Ch or y, 1997 Extragenic suppressors of the Arabidopsis detl mutant identify elements of flowering-time and lightresponse regulatory pathways. Genetics 145: 1125-1137.

Pepper, A., T. Del aney, T. Wash bur n, D. Pool e and J. Chory, 1994 DET 1, a negative regulator of light-mediated development and gene expression in Arabidopsis, encodesa novel nuclear-localized protein. Cell 78: 109-116.

Quail, P. H., 1991 Phytochrome: A light-activated molecular switch that regulates plant gene expression. Annu. Rev. Genet. 25: 389409.

Quail, P. H., W. R. Briggs, J. Chory, R.P. Hangarter, N.P. Harber d etal., 1994 Spotlight on phytochrome nomenclature. Plant Cell 6: 468-471.

Reed, J., and J. Ch or y, 1994 Mutational analyses of light-controlled seedling development in Arabidopsis. Sem. Cell Biol. 5: 327-334.

Reed, J. W., P. Nagpal, D. S. Poole, M. Furuya and J. Chory, 1993 Mutations in the gene for the red/far-red light receptor Phytochrome B alter cell elongation and physiological responses throughout Arabidopsis development. Plant Cell 5: 147-157.

Reed, J. W., A. N agat ani, T. D. El ich, M. Fagan and J. Ch or y, 1994 Phytochrome $A$ and phytochrome $B$ have overlapping but distinct functions in Arabidopsis development. Plant Physiol. 104: 11391149.

Sakamot 0, K., and A. Nagat ani, 1996 Nuclear localization activity of phytochrome B. Plant J. 10: 859-868.

Schneider, K., B. Wel I S, L. Dol an and K. Robert s, 1997 Structural and genetic analysis of epidermal cell differentiation in Arabidopsis primary roots. Development 124: 1789-1798.

Shackl ock, P. S., N. D. Read and A. J. Trewavas, 1992 Cytosolic free calcium mediates red light-induced photomorphogenesis. Nature 358: 753-755.

Sharrock, R. A., and P. H. Quail, 1989 Novel phytochrome sequencesin Arabidopsis thaliana: Structure, evolution, and differential expression of a plant regulatory photoreceptor family. Genes Dev. 3: 1745-1757.

Shinomura, T., A. Nagat ani, J. Chory and M. Fur uya, 1994 The induction of seed germination in Arabidopsis thaliana is regulated principally by phytochrome $B$, and secondarily by phytochrome A. Plant Physiol. 104: 363-371.

Shinomura, T., A. Nagat ani, H. H anzawa, M. Kubota, M. Wat anabe et al., 1996 Action spectra for phytochrome A- and Bspecific photoinduction of seed germination in Arabidopsis thaliana. Proc. Nat. Acad. Sci. USA 93: 8129-8133.

Silverst one, A. L., P. Y. A. Mak, E. C. M artínez, and T.-P. Sun, 1997 Thenew RGA locusencodes a negative regulator of gibberellin response in Arabidopsis thaliana. Genetics 146: 1087-1099.

Smit h, H., 1994 Sensing the light environment: the functions of the phytochrome family, pp. 377-416 in Photomorphogenesis in Plants, edited by R. Kendrick and G. Kr onenber g. Kluwer Academic Publishers, Dordrecht, The Netherlands.

St aub, J. M., N. Wei and X.-W. Deng, 1996 Evidence for FUS6 as a component of the nuclear-localized COP9 complex in Arabidopsis. Plant Cell 8: 2047-2056. 
Szeker es, M., K. N émet h, Z. Koncz-Kal man, J. M at hur, A. Kauschmann et al., 1996 Brassinosteroids rescue the deficiency of CYP90, a cytochrome P450, controlling cell elongation and deetiolation in Arabidopsis. Cell 85: 171-182.

Takahash i, T., A.Gasch, N.Nishizawa and N.-H.Chua, 1995 The DIM INUTO gene of Arabidopsis is involved in regulating cell elongation. Genes Dev. 9: 97-107.

Timpt e, C. S., A. K. Wil son and M. Est ell e, 1992 Effects of the axr2 mutation of Arabidopsis on cell shape in hypocotyl and inflorescence. Planta 188: 271-278.

von Arnim, A. G ., and X.-W. Deng, 1994 Light inactivation of Arabidopsis photomorphogenic repressor COP1 involves a cell-specific regulation of its nucleocytoplasmic partitioning. Cell 79: 10351045.

von Arnim, A., and X.-W. Deng, 1996 Light control of seedling development. Annu. Rev. Plant Physiol. Plant Mol. Biol. 47: 215243.

Wagner, D., U.H oecker and P.H. Quail, 1997 RED1 is necessary for phytochrome B-mediated red light-specific signal transduction in Arabidopsis. Plant Cell 9: 731-743.

Wei, N., D. Chamowit z and X.-W. Deng, 1994a Arabidopsis COP9 is a component of a novel signaling complex mediating light control of development. Cell 78: 117-124.
Wei, N., S. F. Kwok, A. G. von Arnim, A. Lee, T. W. McNell is et al., 1994b Arabidopsis COP8, COP10, and COP11 genes are involved in repression of photomorphogenic development in darkness. Plant Cell 6: 629-643.

Wei, N., and X.-W. Deng, 1996 The role of the COP/DET/FUS genesin light control of Arabidopsisseedling development. Plant Physiol. 112: 871-878.

Whit el am, G. C., E. Johnson, J. Peng, P. Carol, M. L. Anderson et al., 1993 Phytochrome A null mutants of Arabidopsis display a wild-type phenotype in white light. Plant Cell 5: 757-768.

Yeh, K.-C., S.-H. Wu, J. T. Murphy and J. C. Lagarias, 1997 A cyanobacterial phytochrome two-component light sensory system. Science 277: 1505-1508.

Young, J. C., E. Liscum and R. P. H angarter, 1992 Spectral dependence of light-inhibited hypocotyl elongation in photomorphogenic mutants of Arabidopsis: evidence for a UV-A photosensor. Planta 188: 106-114.

Zagotta, M. T., K. A. Hicks, C. I. Jacobs, J. C. Young, R. P. Hangart er et al., 1996 The Arabidopsis EL F3 gene regulates vegetative photomorphogenesis and the photoperiodic induction of flowering. Plant J. 10: 691-702.

Communicating editor: V. Sundar esan 